\title{
Pluripotential theory and convex bodies: large deviation principle
}

\author{
Turgay Bayraktar, Thomas Bloom, Norman Levenberg and Chinh H. Lu
}

\begin{abstract}
We continue the study in [2] in the setting of weighted pluripotential theory arising from polynomials associated to a convex body $P$ in $\left(\mathbb{R}^{+}\right)^{d}$. Our goal is to establish a large deviation principle in this setting specifying the rate function in terms of $P$-pluripotentialtheoretic notions. As an important preliminary step, we first give an existence proof for the solution of a Monge-Ampère equation in an appropriate finite energy class. This is achieved using a variational approach.
\end{abstract}

\section{Introduction}

As in [2], we fix a convex body $P \subset\left(\mathbb{R}^{+}\right)^{d}$ and we define the logarithmic indicator function

$$
H_{P}(z):=\sup _{J \in P} \log \left|z^{J}\right|:=\sup _{\left(j_{1}, \ldots, j_{d}\right) \in P} \log \left[\left|z_{1}\right|^{j_{1}} \ldots\left|z_{d}\right|^{j_{d}}\right] .
$$

We assume throughout that

$$
\Sigma \subset k P \text { for some } k \in \mathbb{Z}^{+}
$$

where

$$
\Sigma:=\left\{\left(x_{1}, \ldots, x_{d}\right) \in \mathbb{R}^{d}: 0 \leq x_{i} \leq 1, \sum_{j=1}^{d} x_{i} \leq 1\right\}
$$

Then

$$
H_{P}(z) \geq \frac{1}{k} \max _{j=1, \ldots, d} \log ^{+}\left|z_{j}\right|
$$

N. Levenberg is supported by Simons Foundation grant No. 354549.

Key words and phrases: convex body, $P$-extremal function, large deviation principle. 2010 Mathematics Subject Classification: 32U15, 32U20, 31C15. 
where $\log ^{+}\left|z_{j}\right|=\max \left[0, \log \left|z_{j}\right|\right]$. We define

$$
L_{P}=L_{P}\left(\mathbb{C}^{d}\right):=\left\{u \in P S H\left(\mathbb{C}^{d}\right): u(z)-H_{P}(z)=O(1),|z| \longrightarrow \infty\right\},
$$

and

$$
L_{P,+}=L_{P,+}\left(\mathbb{C}^{d}\right)=\left\{u \in L_{P}\left(\mathbb{C}^{d}\right): u(z) \geq H_{P}(z)+C_{u}\right\} .
$$

These are generalizations of the classical Lelong classes when $P=\Sigma$. We define the finite-dimensional polynomial spaces

$$
\operatorname{Poly}(n P):=\left\{p(z)=\sum_{J \in n P \cap\left(\mathbb{Z}^{+}\right)^{d}} c_{J} z^{J}: c_{J} \in \mathbb{C}\right\}
$$

for $n=1,2, \ldots$ where $z^{J}=z_{1}^{j_{1}} \ldots z_{d}^{j_{d}}$ for $J=\left(j_{1}, \ldots, j_{d}\right)$. For $p \in \operatorname{Poly}(n P), n \geq 1$ we have $\frac{1}{n} \log |p| \in L_{P}$; also each $u \in L_{P,+}\left(\mathbb{C}^{d}\right)$ is locally bounded in $\mathbb{C}^{d}$. For $P=\Sigma$, we write $\operatorname{Poly}(n P)=\mathcal{P}_{n}$.

Given a compact set $K \subset \mathbb{C}^{d}$, one can define various pluripotential-theoretic notions associated to $K$ related to $L_{P}$ and the polynomial spaces Poly $(n P)$. Our goal in this paper is to prove some probabilistic properties of random point processes on $K$ utilizing these notions and their weighted counterparts. We require an existence proof for the solution of a Monge-Ampère equation in an appropriate finite energy class; this is done in Theorem 2.8 using a variational approach and is of interest on its own. The third section recalls appropriate definitions and properties in $P$-pluripotential theory, mostly following [2]. As in [2], our spaces Poly $(n P)$ do not necessarily arise as holomorphic sections of tensor powers of a line bundle. Subsection 3.3 includes a standard elementary probabilistic result on almost sure convergence of probability measures associated to random arrays on $K$ to a $P$-pluripotential-theoretic equilibrium measure. Section 4 sets up the machinery for the more subtle large deviation principle (LDP), Theorem 5.1, for which we provide two proofs (analogous to those in [9]). As in [9], the first proof was inspired by [6] and the second proof was utilized by Berman in [5]. The reader will find far-reaching applications and interpretations of LDP's in the appropriate settings of holomorphic line bundles over a compact, complex manifold in [5]. In particular, the case where $P$ is a convex integral polytope (vertices in $\mathbb{Z}^{d}$ ) which is the moment polytope for a toric manifold ( $P$ is Delzant) is covered in [5].

\section{Monge-Ampère and $P$-pluripotential theory}

\subsection{Monge-Ampère equations with prescribed singularity}

In this section, $(X, \omega)$ is a compact Kähler manifold of dimension $d$. 


\subsubsection{Quasi-plurisubharmonic functions}

A function $u: X \rightarrow \mathbb{R} \cup\{-\infty\}$ is called quasi-plurisubharmonic (quasi-psh) if locally $u=\rho+\varphi$, where $\varphi$ is plurisubharmonic and $\rho$ is smooth.

We let $P S H(X, \omega)$ denote the set of $\omega$-psh functions, i.e. quasi-psh functions $u$ such that $\omega_{u}:=\omega+d d^{c} u \geq 0$ in the sense of currents on $X$.

Given $u, v \in P S H(X, \omega)$ we say that $u$ is more singular than $v$ (and we write $u \prec v)$ if $u \leq v+C$ on $X$, for some constant $C$. We say that $u$ has the same singularity as $v$ (and we write $u \simeq v$ ) if $u \prec v$ and $v \prec u$.

Given $\phi \in P S H(X, \omega)$, we let $P S H(X, \omega, \phi)$ denote the set of $\omega$-psh functions $u$ which are more singular than $\phi$.

\subsubsection{Nonpluripolar Monge-Ampère measure}

For bounded $\omega$-psh functions $u_{1}, \ldots, u_{d}$, the Monge-Ampère product $(\omega+$ $\left.d d^{c} u_{1}\right) \wedge \ldots \wedge\left(\omega+d d^{c} u_{d}\right)$ is well-defined as a positive Radon measure on $X$ (see [14], [3]). For general $\omega$-psh functions $u_{1}, \ldots, u_{d}$, the sequence of positive measures

$$
\mathbf{1}_{\cap\left\{u_{j}>-k\right\}}\left(\omega+d d^{c} \max \left(u_{1},-k\right)\right) \wedge \ldots \wedge\left(\omega+d d^{c} \max \left(u_{d},-k\right)\right)
$$

is non-decreasing in $k$ and the limiting measure, which is called the nonpluripolar product of $\omega_{u_{1}}, \ldots, \omega_{u_{d}}$, is denoted by

$$
\omega_{u_{1}} \wedge \ldots \wedge \omega_{u_{d}} .
$$

When $u_{1}=\ldots=u_{d}=u$ we write $\omega_{u}^{d}:=\omega_{u} \wedge \ldots \wedge \omega_{u}$. Note that by definition $\int_{X} \omega_{u_{1}} \wedge$ $\ldots \wedge \omega_{u_{d}} \leq \int_{X} \omega^{d}$.

It was proved in [20, Theorem 1.2] and [11, Theorem 1.1] that the total mass of nonpluripolar Monge-Ampère products is decreasing with respect to singularity type. More precisely,

Theorem 2.1. Let $\omega_{1}, \ldots, \omega_{d}$ be Kähler forms on $X$. If $u_{j} \prec v_{j}, j=1, \ldots, d$, are $\omega_{j}$-psh functions then

$$
\int_{X}\left(\omega_{1}+d d^{c} u_{1}\right) \wedge \ldots \wedge\left(\omega_{d}+d d^{c} u_{d}\right) \leq \int_{X}\left(\omega_{1}+d d^{c} v_{1}\right) \wedge \ldots \wedge\left(\omega_{d}+d d^{c} v_{d}\right) .
$$

As noted above, for a general $\omega$-psh function $u$ we have the estimate $\int_{X} \omega_{u}^{d} \leq$ $\int_{X} \omega^{d}$. Following [15] we let $\mathcal{E}(X, \omega)$ denote the set of all $\omega$-psh functions with maximal total mass, i.e.

$$
\mathcal{E}(X, \omega):=\left\{u \in \operatorname{PSH}(X, \omega): \int_{X} \omega_{u}^{d}=\int_{X} \omega^{d}\right\} .
$$


Given $\phi \in P S H(X, \omega)$, we define

$$
\mathcal{E}(X, \omega, \phi):=\left\{u \in \operatorname{PSH}(X, \omega, \phi): \int_{X} \omega_{u}^{d}=\int_{X} \omega_{\phi}^{d}\right\} .
$$

Proposition 2.2. Let $\phi \in P S H(X, \omega)$. The following are equivalent:

(1) $\mathcal{E}(X, \omega, \phi) \cap \mathcal{E}(X, \omega) \neq \varnothing$;

(2) $\phi \in \mathcal{E}(X, \omega)$;

(3) $\mathcal{E}(X, \omega, \phi) \subset \mathcal{E}(X, \omega)$.

Proof. We first prove $(1) \Longrightarrow(2)$. If $u \in \mathcal{E}(X, \omega, \phi) \cap \mathcal{E}(X, \omega)$ then $\int_{X} \omega_{u}^{d}=\int_{X} \omega^{d}$. On the other hand, since $u$ is more singular than $\phi$, Theorem 2.1 ensures that

$$
\int_{X} \omega^{d}=\int_{X} \omega_{u}^{d} \leq \int_{X} \omega_{\phi}^{d} \leq \int_{X} \omega^{d}
$$

hence equality holds, proving that $\phi \in \mathcal{E}(X, \omega)$.

Now we prove $(2) \Longrightarrow(3)$. If $\phi \in \mathcal{E}(X, \omega)$ and $u \in \mathcal{E}(X, \omega, \phi)$ then

$$
\int_{X} \omega_{u}^{d}=\int_{X} \omega_{\phi}^{d}=\int_{X} \omega^{d}
$$

hence $u \in \mathcal{E}(X, \omega)$.

Finally $(3) \Longrightarrow(1)$ is obvious.

Proposition 2.3. Assume that $\phi_{j} \in P S H\left(X, \omega_{j}\right), j=1, \ldots, d$ with $\int_{X}\left(\omega_{j}+\right.$ $\left.d d^{c} \phi_{j}\right)^{d}>0$. If $u_{j} \in \mathcal{E}\left(X, \omega_{j}, \phi_{j}\right), j=1, \ldots, d$, then

$$
\int_{X}\left(\omega_{1}+d d^{c} u_{1}\right) \wedge \ldots \wedge\left(\omega_{d}+d d^{c} u_{d}\right)=\int_{X}\left(\omega_{1}+d d^{c} \phi_{1}\right) \wedge \ldots \wedge\left(\omega_{d}+d d^{c} \phi_{d}\right) .
$$

Proof. Theorem 2.1 gives one inequality. The other one follows from [11, Proposition 3.1 and Theorem 3.14].

\subsubsection{Model potentials}

For a function $f: X \rightarrow \mathbb{R} \cup\{-\infty\}$, we let $f^{*}$ denote its uppersemicontinuous (usc) regularization, i.e.

$$
f^{*}(x):=\limsup _{X \ni y \rightarrow x} f(y) .
$$

Given $\phi \in P S H(X, \omega)$, following J. Ross and D. Witt Nyström [18], we define

$$
P_{\omega}[\phi]:=\left(\lim _{t \rightarrow+\infty} P_{\omega}(\min (\phi+t, 0))\right)^{*} .
$$


Here, for a function $f, P_{\omega}(f)$ is defined as

$$
P_{\omega}(f):=(x \longmapsto \sup \{u(x): u \in P S H(X, \omega), u \leq f\})^{*} .
$$

It was shown in [11, Theorem 3.8] that the nonpluripolar Monge-Ampère measure of $P_{\omega}[\phi]$ is dominated by Lebesgue measure:

$$
\left(\omega+d d^{c} P_{\omega}[\phi]\right)^{d} \leq \mathbf{1}_{\left\{P_{\omega}[\phi]=0\right\}} \omega^{d} \leq \omega^{d} .
$$

This fact plays a crucial role in solving the complex Monge-Ampère equation. For the reader's convenience, we note that in the notation of [11] (on the left)

$$
P_{[\omega, \phi]}(0)=P_{\omega}[\phi] .
$$

Definition 2.4. A function $\phi \in P S H(X, \omega)$ is called a model potential if $\int_{X} \omega_{\phi}^{d}>$ 0 and $P_{\omega}[\phi]=\phi$. A function $u \in P S H(X, \omega)$ has model type singularity if $u$ has the same singularity as $P_{\omega}[u]$; i.e., $u-P_{\omega}[u]$ is bounded on $X$.

There are plenty of model potentials. If $\varphi \in P S H(X, \omega)$ with $\int_{X} \omega_{\varphi}^{d}>0$ then, by [11, Theorem 3.12], $P_{\omega}[\varphi]$ is a model potential. In particular, if $\int_{X} \omega_{\varphi}^{d}=\int_{X} \omega^{d}$ (i.e. $\varphi \in \mathcal{E}(X, \omega))$ then $P_{\omega}[\varphi]=0$.

We will use the following property of model potentials proved in [11, Theorem 3.12]: if $\phi$ is a model potential then

$$
u \in P S H(X, \omega, \phi) \Longrightarrow u-\sup _{X} u \leq \phi
$$

In the sequel we always assume that $\phi$ has model type singularity and small unbounded locus; i.e., $\phi$ is locally bounded outside a closed complete pluripolar set, allowing us to use the variational approach of [7] as explained in [11].

\subsubsection{The variational approach}

We call a measure which puts no mass on pluripolar sets a nonpluripolar measure. For a positive nonpluripolar measure $\mu$ on $X$ we let $L_{\mu}$ denote the following linear functional on $\operatorname{PSH}(X, \omega, \phi)$ :

$$
L_{\mu}(u):=\int_{X}(u-\phi) d \mu .
$$

For $u \in P S H(X, \omega)$ with $u \simeq \phi$, we define the Monge-Ampère energy

$$
\mathbf{E}_{\phi}(u):=\frac{1}{(d+1)} \sum_{k=0}^{d} \int_{X}(u-\phi) \omega_{u}^{k} \wedge \omega_{\phi}^{d-k} .
$$


It was shown in [11, Theorem 4.10] (by adapting the arguments of [7]) that $\mathbf{E}_{\phi}$ is non-decreasing and concave along affine curves, giving rise to its trivial extension to $\operatorname{PSH}(X, \omega, \phi)$.

We define

$$
\mathcal{E}^{1}(X, \omega, \phi):=\left\{u \in P S H(X, \omega, \phi): \mathbf{E}_{\phi}(u)>-\infty\right\} .
$$

The following criterion was proved in [11, Theorem 4.13]:

Proposition 2.5. Let $u \in P S H(X, \omega, \phi)$. Then $u \in \mathcal{E}^{1}(X, \omega, \phi)$ iff $u \in \mathcal{E}(X, \omega, \phi)$ and $\int_{X}(u-\phi) \omega_{u}^{d}>-\infty$.

Lemma 2.6. If $E$ is pluripolar then there exists $u \in \mathcal{E}^{1}(X, \omega, \phi)$ such that $E \subset$ $\{u=-\infty\}$.

Proof. Without loss of generality we can assume that $\phi$ is a model potential. Then (2.1) gives $\int_{X}|\phi| \omega_{\phi}^{d}=0$. It follows from [7, Corollary 2.11] that there exists $v \in \mathcal{E}^{1}(X, \omega, 0), v \leq 0$, such that $E \subset\{v=-\infty\}$. Set $u:=P_{\omega}(\min (v, \phi))$. Then $E \subset\{u=$ $-\infty\}$ and we claim that $u \in \mathcal{E}^{1}(X, \omega, \phi)$. For each $j \in \mathbb{N}$ we set $v_{j}:=\max (v,-j)$ and $u_{j}:=P_{\omega}\left(\min \left(v_{j}, \phi\right)\right)$. Then $u_{j}$ decreases to $u$ and $u_{j} \simeq \phi$. Using [11, Theorem 4.10 and Lemma 4.15] it suffices to check that $\left\{\int_{X}\left|u_{j}-\phi\right| \omega_{u_{j}}^{d}\right\}$ is uniformly bounded. It follows from [11, Lemma 3.7] that

$$
\begin{aligned}
\int_{X}\left|u_{j}-\phi\right| \omega_{u_{j}}^{d} \leq \int_{X}\left|u_{j}\right| \omega_{u_{j}}^{d} & \leq \int_{X}\left|v_{j}\right| \omega_{v_{j}}^{d}+\int_{X}|\phi| \omega_{\phi}^{d} \\
& =\int_{X}\left|v_{j}\right| \omega_{v_{j}}^{d} .
\end{aligned}
$$

The fact that $\int_{X}\left|v_{j}\right| \omega_{v_{j}}^{d}$ is uniformly bounded follows from [15, Corollary 2.4] since $v \in \mathcal{E}^{1}(X, \omega, 0)$. This concludes the proof.

Lemma 2.7. Assume that $\mathcal{E}^{1}(X, \omega, \phi) \subset L^{1}(X, \mu)$. Then, for each $C>0, L_{\mu}$ is bounded on

$$
E_{C}:=\left\{u \in P S H(X, \omega, \phi): \sup _{X} u \leq 0 \text { and } \mathbf{E}_{\phi}(u) \geq-C\right\} .
$$

Proof. By concavity of $\mathbf{E}_{\phi}$ the set $E_{C}$ is convex. We now show that $E_{C}$ is compact in the $L^{1}\left(X, \omega^{d}\right)$ topology. Let $\left\{u_{j}\right\}$ be a sequence in $E_{C}$. We claim that $\left\{\sup _{X} u_{j}\right\}$ is bounded. Indeed, by [11, Theorem 4.10]

$$
\begin{aligned}
\mathbf{E}_{\phi}\left(u_{j}\right) & \leq \int_{X}\left(u_{j}-\phi\right) \omega_{\phi}^{d} \\
& \leq\left(\sup _{X} u_{j}\right) \int_{X} \omega_{\phi}^{d}+\int_{X}\left(u_{j}-\sup _{X} u_{j}-\phi\right) \omega_{\phi}^{d} .
\end{aligned}
$$


It follows from $(2.2)$ that $u_{j}-\sup _{X} u_{j} \leq P_{\omega}[\phi] \leq \phi+C_{0}$, where $C_{0}$ is a constant. The boundedness of $\left\{\sup _{X} u_{j}\right\}$ then follows from that of $\left\{\mathbf{E}_{\phi}\left(u_{j}\right)\right\}$ and the above estimate. This proves the claim.

A subsequence of $\left\{u_{j}\right\}$, still denoted by $\left\{u_{j}\right\}$, converges in $L^{1}\left(X, \omega^{d}\right)$ to $u \in$ $P S H(X, \omega)$ with $\sup _{X} u \leq 0$. Since $u_{j}-\sup _{X} u_{j} \leq \phi+C_{0}$, we have $u-\sup _{X} u \leq \phi+$ $C_{0}$. This proves that $u \in P S H(X, \omega, \phi)$. The upper semicontinuity of $\mathbf{E}_{\phi}$ (see [11, Proposition 4.19]) ensures that $\mathbf{E}_{\phi}(u) \geq-C$, hence $u \in E_{C}$. This proves that $E_{C}$ is compact in the $L^{1}\left(X, \omega^{d}\right)$ topology.

The result then follows from [7, Proposition 3.4].

The goal of this section is to prove the following result:

Theorem 2.8. Assume that $\mu$ is a nonpluripolar positive measure on $X$ such that $\mu(X)=\int_{X} \omega_{\phi}^{d}$. The following are equivalent

(1) $\mu$ has finite energy, i.e., $L_{\mu}$ is finite on $\mathcal{E}^{1}(X, \omega, \phi)$;

(2) there exists $u \in \mathcal{E}^{1}(X, \omega, \phi)$ such that $\omega_{u}^{d}=\mu$;

(3) there exists a unique $u \in \mathcal{E}^{1}(X, \omega, \phi)$ such that

$$
F_{\mu}(u)=\max _{v \in \mathcal{E}^{1}(X, \omega, \phi)} F_{\mu}(v)<+\infty
$$

where $F_{\mu}=\mathbf{E}_{\phi}-L_{\mu}$.

Remark 2.9. It was shown in [11, Theorem 4.28] that a unique (normalized) solution $u$ in $\mathcal{E}(X, \omega, \phi)$ always exists (without the finite energy assumption on $\mu$ ). But that proof does not give a solution in $\mathcal{E}^{1}(X, \omega, \phi)$. Below, we will follow the proof of [11, Theorem 4.28] and use the finite energy condition, $\mathcal{E}^{1}(X, \omega, \phi) \subset L^{1}(X, \mu)$, to prove that $u$ belongs to $\mathcal{E}^{1}(X, \omega, \phi)$.

Lemma 2.10. Assume that $\mathcal{E}^{1}(X, \omega, \phi) \subset L^{1}(X, \mu)$. Then there exists a positive constant $C$ such that, for all $u \in \mathcal{E}^{1}(X, \omega, \phi)$ with $\sup _{X} u=0$,

$$
L_{\mu}(u) \geq-C\left(1+\left|\mathbf{E}_{\phi}(u)\right|^{1 / 2}\right) .
$$

The proof below uses ideas in [7], [15].

Proof. Since $\phi$ has model type singularity, it follows from [11, Theorem 4.10] that $\mathbf{E}_{\phi}-\mathbf{E}_{P_{\omega}[\phi]}$ is bounded. Without loss of generality we can assume in this proof that $\phi=P_{\omega}[\phi]$. Fix $u \in \mathcal{E}^{1}(X, \omega, \phi)$ such that $\sup _{X} u=0$ and $\left|\mathbf{E}_{\phi}(u)\right|>1$. Then, by [11, Theorem 3.12], $u \leq \phi$. Set $a=\left|\mathbf{E}_{\phi}(u)\right|^{-1 / 2} \in(0,1)$, and $v:=a u+(1-a) \phi \in$ 
$\mathcal{E}^{1}(X, \omega, \phi)$. We estimate $\mathbf{E}_{\phi}(v)$ as follows

$$
\begin{aligned}
(d+1) \mathbf{E}_{\phi}(v) & =a \sum_{k=0}^{d} \int_{X}(u-\phi) \omega_{v}^{k} \wedge \omega_{\phi}^{d-k} \\
& =a \sum_{k=0}^{d} \int_{X}(u-\phi)\left(a \omega_{u}+(1-a) \omega_{\phi}\right)^{k} \wedge \omega_{\phi}^{d-k} \\
& \geq C(d) a \int_{X}(u-\phi) \omega_{\phi}^{d}+C(d) a^{2} \sum_{k=0}^{d} \int_{X}(u-\phi) \omega_{u}^{k} \wedge \omega_{\phi}^{d},
\end{aligned}
$$

where $C(d)$ is a positive constant which only depends on $d$. It follows from $\phi=P_{\omega}[\phi]$ and [11, Theorem 3.8] that $\omega_{\phi}^{d} \leq \omega^{d}$ (recall (2.1)). This together with [14, Proposition 2.7] give

$$
\int_{X}(u-\phi) \omega_{\phi}^{d} \geq-C_{1}
$$

for a uniform constant $C_{1}$. Therefore,

$$
(d+1) \mathbf{E}_{\phi}(v) \geq-C_{1} C(d) a+C_{2} a^{2} \mathbf{E}_{\phi}(u) \geq-C_{3} .
$$

It thus follows from Lemma 2.7 that $L_{\mu}(v) \geq-C_{4}$ for a uniform constant $C_{4}>0$. Thus

$$
\int_{X}(u-\phi) d \mu \geq-C_{4} / a
$$

which gives (2.5).

We are now ready to prove Theorem 2.8.

Proof of Theorem 2.8. Without loss of generality we can assume that $\phi$ is a model potential. We first prove $(1) \Longrightarrow(2)$. We write $\mu=f \nu$, where $\nu$ is a nonpluripolar positive measure satisfying, for all Borel subsets $B \subset X$,

$$
\nu(B) \leq A \operatorname{Cap}_{\phi}(B),
$$

for some positive constant $A$, and $0 \leq f \in L^{1}(X, \nu)$ (cf., [11, Lemma 4.26]). Here $\mathrm{Cap}_{\phi}$ is defined as

$$
\operatorname{Cap}_{\phi}(B):=\sup \left\{\int_{B} \omega_{u}^{d}: u \in P S H(X, \omega), \phi-1 \leq u \leq \phi\right\} .
$$

Set, for $k \in \mathbb{N}, \mu_{k}:=c_{k} \min (f, k) \nu$ where $c_{k}>0$ is chosen so that $\mu_{k}(X)=\int_{X} \omega_{\phi}^{d}$; this is needed in order to solve the Monge-Ampère equation in the class $\mathcal{E}^{1}(X, \omega, \phi)$. 
For $k$ large enough, $1 \leq c_{k} \leq 2$ and $c_{k} \rightarrow 1$ as $k \rightarrow+\infty$. It follows from [11, Theorem 4.25] that there exists $u_{j} \in \mathcal{E}^{1}(X, \omega, \phi), \sup _{X} u_{j}=0$, such that $\omega_{u_{j}}^{d}=\mu_{j}$; by $[11$, Theorem 3.12], $u_{j} \leq \phi$. A subsequence of $\left\{u_{j}\right\}$ which, by abuse of notation, will be denoted by $\left\{u_{j}\right\}$, converges in $L^{1}\left(X, \omega^{d}\right)$ to $u \in P S H(X, \omega)$ with $u \leq \phi$. Define $v_{k}:=\left(\sup _{j \geq k} u_{j}\right)^{*}$. Then $v_{k} \searrow u$ and $\sup _{X} v_{k}=0$. It follows from (2.5) and [11, Theorem 4.10 ] that

$$
\begin{aligned}
\left|\mathbf{E}_{\phi}\left(u_{j}\right)\right| & \leq \int_{X}\left|u_{j}-\phi\right| \omega_{u_{j}}^{d} \leq 2 \int_{X}\left|u_{j}-\phi\right| d \mu \\
& \leq 2 C\left(1+\left|\mathbf{E}_{\phi}\left(u_{j}\right)\right|^{1 / 2}\right) .
\end{aligned}
$$

Therefore $\left\{\left|\mathbf{E}_{\phi}\left(u_{j}\right)\right|\right\}$ is bounded, hence so is $\left\{\left|\mathbf{E}_{\phi}\left(v_{j}\right)\right|\right\}$ since $\mathbf{E}_{\phi}$ is non-decreasing. It then follows from [11, Lemma 4.15] that $u \in \mathcal{E}^{1}(X, \omega, \phi)$.

Now, repeating the arguments of $\left[11\right.$, Theorem 4.28] we can show that $\omega_{u}^{d}=\mu$, finishing the proof of $(1) \Longrightarrow(2)$.

We next prove $(2) \Longrightarrow(3)$. Assume that $\mu=\omega_{u}^{d}$ for some $u \in \mathcal{E}^{1}(X, \omega, \phi)$. For all $v \in \mathcal{E}^{1}(X, \omega, \phi)$, by [11, Theorem 4.10] and Proposition 2.5 we have

$$
\begin{aligned}
L_{\mu}(v) & =\int_{X}(v-\phi) \omega_{u}^{d} \\
& =\int_{X}(v-u) \omega_{u}^{d}+\int_{X}(u-\phi) \omega_{u}^{d} \\
& \geq \mathbf{E}_{\phi}(v)-\mathbf{E}_{\phi}(u)+\int_{X}(u-\phi) \omega_{u}^{d}>-\infty .
\end{aligned}
$$

Hence $L_{\mu}$ is finite on $\mathcal{E}^{1}(X, \omega, \phi)$. Now, for all $v \in \mathcal{E}^{1}(X, \omega, \phi)$, by [11, Theorem 4.10] we have

$$
F_{\mu}(v)-F_{\mu}(u)=\mathbf{E}_{\phi}(v)-\mathbf{E}_{\phi}(u)-\int_{X}(v-u) \omega_{u}^{d} \leq 0 .
$$

This gives (3). Finally, $(3) \Longrightarrow(1)$ is obvious.

\subsection{Monge-Ampère equations on $\mathbb{C}^{d}$ with prescribed growth}

As in the introduction we let $P$ be a convex body contained in $\left(\mathbb{R}^{+}\right)^{d}$ and fix $r>0$ such that $P \subset r \Sigma$. We assume (1.2); i.e., $\Sigma \subset k P$ for some $k \in \mathbb{Z}^{+}$. This ensures that $H_{P}$ in (1.1) is locally bounded on $\mathbb{C}^{d}$ (and of course $H_{P} \in L_{P}^{+}\left(\mathbb{C}^{d}\right)$ ). Let $u \in L_{P}\left(\mathbb{C}^{d}\right)$ and define

$$
\tilde{u}(z):=u(z)-\frac{r}{2} \log \left(1+|z|^{2}\right), z \in \mathbb{C}^{d} .
$$


Consider the projective space $\mathbb{P}^{d}$ equipped with the Kähler metric $\omega:=r \omega_{F S}$, where

$$
\omega_{F S}=d d^{c} \frac{1}{2} \log \left(1+|z|^{2}\right)
$$

on $\mathbb{C}^{d}$. Then $\tilde{u}$ is bounded from above on $\mathbb{C}^{d}$. It thus can be extended to $\mathbb{P}^{d}$ as a function in $P S H\left(\mathbb{P}^{d}, \omega\right)$.

For a plurisubharmonic function $u$ on $\mathbb{C}^{d}$, we let $\left(d d^{c} u\right)^{d}$ denote its nonpluripolar Monge-Ampère measure; i.e., $\left(d d^{c} u\right)^{d}$ is the increasing limit of the sequence of measures $\mathbf{1}_{\{u>-k\}}\left(d d^{c} \max (u,-k)\right)^{d}$. Then

$$
\omega_{\tilde{u}}^{d}=\left(\omega+d d^{c} \tilde{u}\right)^{d}=\left(d d^{c} u\right)^{d} \text { on } \mathbb{C}^{d} .
$$

If $u \in L_{P}\left(\mathbb{C}^{d}\right)$ then

$$
\int_{\mathbb{C}^{d}}\left(d d^{c} u\right)^{d} \leq \int_{\mathbb{C}^{d}}\left(d d^{c} H_{P}\right)^{d}=d ! \operatorname{Vol}(P)=: \gamma_{d}=\gamma_{d}(P)
$$

(cf., equation (2.4) in [2]). We define

$$
\mathcal{E}_{P}\left(\mathbb{C}^{d}\right):=\left\{u \in L_{P}\left(\mathbb{C}^{d}\right): \int_{\mathbb{C}^{d}}\left(d d^{c} u\right)^{d}=\gamma_{d}\right\}
$$

By the construction in $(2.6)$ we have that $\widetilde{H}_{P} \in P S H\left(\mathbb{P}^{d}, \omega\right)$. We define

$$
\widetilde{\Phi}_{P}:=P_{\omega}\left[\widetilde{H}_{P}\right] \text {. }
$$

The key point here, which follows from [12, Theorem 7.2], is that $\widetilde{H}_{P}$ has model type singularity (recall Definition 2.4) and hence the same singularity as $\widetilde{\Phi}_{P}$. Defining $\Phi_{P}$ on $\mathbb{C}^{d}$ using $(2.6)$; i.e., for $z \in \mathbb{C}^{d}$,

$$
\Phi_{P}(z)=\widetilde{\Phi}_{P}(z)+\frac{r}{2} \log \left(1+|z|^{2}\right),
$$

we thus have $\Phi_{P} \in L_{P,+}\left(\mathbb{C}^{d}\right)$. The advantage of using $\Phi_{P}$ is that, by $(2.1)$, $\left(d d^{c} \Phi_{P}\right)^{d} \leq \omega^{d}$ on $\mathbb{C}^{d}$. Note that $L_{P,+}\left(\mathbb{C}^{d}\right) \subset \mathcal{E}_{P}\left(\mathbb{C}^{d}\right)$. For $u, v \in L_{P}^{+}\left(\mathbb{C}^{d}\right)$ we define

$$
E_{v}(u):=\frac{1}{(d+1)} \sum_{j=0}^{d} \int_{\mathbb{C}^{d}}(u-v)\left(d d^{c} u\right)^{j} \wedge\left(d d^{c} v\right)^{d-j}
$$

The corresponding global energy (see (2.3)) is defined as

$$
\mathbf{E}_{\tilde{v}}(\tilde{u}):=\frac{1}{(d+1)} \sum_{j=0}^{d} \int_{\mathbb{P}^{d}}(\tilde{u}-\tilde{v})\left(\omega+d d^{c} \tilde{u}\right)^{j} \wedge\left(\omega+d d^{c} \tilde{v}\right)^{d-j} .
$$


Then $E_{v}$ is non-decreasing and concave along affine curves in $L_{P,+}\left(\mathbb{C}^{d}\right)$. We extend $E_{v}$ to $L_{P}\left(\mathbb{C}^{d}\right)$ in an obvious way. Note that $E_{v}$ may take the value $-\infty$. We define

$$
\mathcal{E}_{P}^{1}\left(\mathbb{C}^{d}\right):=\left\{u \in L_{P}\left(\mathbb{C}^{d}\right): E_{H_{P}}(u)>-\infty\right\} .
$$

We observe that in the above definition we can replace $E_{H_{P}}$ by $E_{\Phi_{P}}$, since for $u \in L_{P,+}\left(\mathbb{C}^{d}\right)$, by the cocycle property (cf. Proposition $3.3[2]$ ),

$$
E_{H_{P}}(u)-E_{H_{P}}\left(\Phi_{P}\right)=E_{\Phi_{P}}(u) .
$$

We thus have the following important identification (see (2.4)):

$$
u \in \mathcal{E}_{P}^{1}\left(\mathbb{C}^{d}\right) \Longleftrightarrow \tilde{u} \in \mathcal{E}^{1}\left(\mathbb{P}^{d}, \omega, \widetilde{\Phi}_{P}\right) .
$$

We then have the following local version of Proposition 2.5:

Proposition 2.11. Let $u \in L_{P}\left(\mathbb{C}^{d}\right)$. Then $u \in \mathcal{E}_{P}^{1}\left(\mathbb{C}^{d}\right)$ iff $u \in \mathcal{E}_{P}\left(\mathbb{C}^{d}\right)$ and $\int_{\mathbb{C}^{d}}\left(u-H_{P}\right)\left(d d^{c} u\right)^{d}>-\infty$. In particular, if $\operatorname{supp}\left(d d^{c} u\right)^{d}$ is compact, $u \in \mathcal{E}_{P}^{1}\left(\mathbb{C}^{d}\right)$ iff $\int_{\mathbb{C}^{d}}\left(d d^{c} u\right)^{d}=\gamma_{d}$ and $\int_{\mathbb{C}^{d}} u\left(d d^{c} u\right)^{d}>-\infty$.

Proof. Since $\widetilde{H}_{P} \simeq \widetilde{\Phi}_{P}$,

$$
\int_{\mathbb{P}^{d}}\left(\tilde{u}-\widetilde{H}_{P}\right) \omega_{\tilde{u}}^{d}>-\infty \text { iff } \int_{\mathbb{P}^{d}}\left(\tilde{u}-\widetilde{\Phi}_{P}\right) \omega_{\tilde{u}}^{d}>-\infty
$$

where $\tilde{u} \in P S H\left(\mathbb{P}^{d}, \omega\right)$ and $u$ are related by (2.6). Moreover, $\Phi_{P} \in L_{P,+}\left(\mathbb{C}^{d}\right)$ implies $u \leq \Phi_{P}+C$ so that $\tilde{u} \in P S H\left(\mathbb{P}^{d}, \omega, \widetilde{\Phi}_{P}\right)$. But

$$
\int_{\mathbb{P}^{d}}\left(\tilde{u}-\widetilde{H}_{P}\right) \omega_{\tilde{u}}^{d}=\int_{\mathbb{C}^{d}}\left(u-H_{P}\right)\left(d d^{c} u\right)^{d}
$$

and the result follows from (2.8) by applying Proposition 2.5 to $\tilde{u}$. For the last statement, note that for general $u \in L_{P}\left(\mathbb{C}^{d}\right)$ we may have $\int_{\mathbb{C}^{d}} H_{P}\left(d d^{c} u\right)^{d}=+\infty$, but if $\left(d d^{c} u\right)^{d}$ has compact support then $\int_{\mathbb{C}^{d}} H_{P}\left(d d^{c} u\right)^{d}$ is finite.

Note that Theorem 2.1 and Proposition 2.3 give the following result:

Theorem 2.12. Let $u_{1}, \ldots, u_{d}$ be functions in $\mathcal{E}_{P}\left(\mathbb{C}^{d}\right)$. Then

$$
\int_{\mathbb{C}^{d}} d d^{c} u_{1} \wedge \ldots \wedge d d^{c} u_{d}=\gamma_{d}
$$

For $u_{1}, \ldots, u_{n} \in L_{P,+}\left(\mathbb{C}^{d}\right)$ Theorem 2.12 was proved in [1, Proposition 2.7].

Having the correspondence (2.8) we can state a local version of Theorem 2.8; this will be used in the sequel. Let $\mathcal{M}_{P}\left(\mathbb{C}^{d}\right)$ denote the set of all positive Borel measures $\mu$ on $\mathbb{C}^{d}$ with $\mu\left(\mathbb{C}^{d}\right)=d ! \operatorname{Vol}(P)=\gamma_{d}$. 
Theorem 2.13. Assume that $\mu \in \mathcal{M}_{P}\left(\mathbb{C}^{d}\right)$ is a positive nonpluripolar Borel measure. The following are equivalent

(1) $\mathcal{E}_{P}^{1}\left(\mathbb{C}^{d}\right) \subset L^{1}\left(\mathbb{C}^{d}, \mu\right)$

(2) there exists $u \in \mathcal{E}_{P}^{1}\left(\mathbb{C}^{d}\right)$ such that $\left(d d^{c} u\right)^{d}=\mu$;

(3) there exists $u \in \mathcal{E}_{P}^{1}\left(\mathbb{C}^{d}\right)$ such that

$$
\mathcal{F}_{\mu}(u)=\max _{v \in \mathcal{E}_{P}^{1}\left(\mathbb{C}^{d}\right)} \mathcal{F}_{\mu}(v)<+\infty
$$

A priori the functional $\mathcal{F}_{\mu}$ is defined for $u \in \mathcal{E}_{P}^{1}\left(\mathbb{C}^{d}\right)$ by

$$
\mathcal{F}_{\mu, \Phi_{P}}(u):=E_{\Phi_{P}}(u)-\int_{\mathbb{C}^{d}}\left(u-\Phi_{P}\right) d \mu .
$$

However, using this notation, since

$$
\mathcal{F}_{\mu, \Phi_{P}}(u)-\mathcal{F}_{\mu, H_{P}}(u)=\mathcal{F}_{\mu, \Phi_{P}}\left(H_{P}\right)
$$

in statement (3) of Theorem 2.13 we can take either of the two definitions $\mathcal{F}_{\mu, \Phi_{P}}$ or $\mathcal{F}_{\mu, H_{P}}$ for $\mathcal{F}_{\mu}$.

Remark 2.14. If $\mu$ has compact support in $\mathbb{C}^{d}$ then $\int_{\mathbb{C}^{d}} \Phi_{P} d \mu$ and $\int_{\mathbb{C}^{d}} H_{P} d \mu$ are finite. Therefore, the functional $\mathcal{F}_{\mu}$ can be replaced by

$$
u \longmapsto E_{H_{P}}(u)-\int_{\mathbb{C}^{d}} u d \mu .
$$

Using the remark, for $\mu \in \mathcal{M}_{P}\left(\mathbb{C}^{d}\right)$ with compact support, it is natural to define the Legendre-type transform of $E_{H_{P}}$ :

$$
E^{*}(\mu):=\sup _{u \in \mathcal{E}_{P}^{1}\left(\mathbb{C}^{d}\right)}\left[E_{H_{P}}(u)-\int_{\mathbb{C}^{d}} u d \mu\right]
$$

This functional, which will appear in the rate function for our LDP, will be given a more concrete interpretation using $P$-pluripotential theory in section 4 ; cf., equation (4.18).

Finally, for future use, we record the following consequence of Lemma 2.6 and the correspondence (2.8).

Lemma 2.15. If $E \subset \mathbb{C}^{d}$ is pluripolar then there exists $u \in \mathcal{E}_{P}^{1}\left(\mathbb{C}^{d}\right)$ such that $E \subset\{u=-\infty\}$. 


\section{3. $P$-pluripotential theory notions}

Given $E \subset \mathbb{C}^{d}$, the $P$-extremal function of $E$ is

$$
V_{P, E}^{*}(z):=\limsup _{\zeta \rightarrow z} V_{P, E}(\zeta)
$$

where

$$
V_{P, E}(z):=\sup \left\{u(z): u \in L_{P}\left(\mathbb{C}^{d}\right), u \leq 0 \text { on } E\right\}
$$

For $K \subset \mathbb{C}^{d}$ compact, $w: K \rightarrow \mathbb{R}^{+}$is an admissible weight function on $K$ if $w \geq 0$ is an uppersemicontinuous function with $\{z \in K: w(z)>0\}$ nonpluripolar. Setting $Q:=-\log w$, we write $Q \in \mathcal{A}(K)$ and define the weighted $P$-extremal function

$$
V_{P, K, Q}^{*}(z):=\limsup _{\zeta \rightarrow z} V_{P, K, Q}(\zeta)
$$

where

$$
V_{P, K, Q}(z):=\sup \left\{u(z): u \in L_{P}\left(\mathbb{C}^{d}\right), u \leq Q \text { on } K\right\} .
$$

If $Q=0$ we write $V_{P, K, Q}=V_{P, K}$, consistent with the previous notation. For $P=\Sigma$,

$$
V_{\Sigma, K, Q}(z)=V_{K, Q}(z):=\sup \left\{u(z): u \in L\left(\mathbb{C}^{d}\right), u \leq Q \text { on } K\right\}
$$

is the usual weighed extremal function as in Appendix B of [19].

We write (omitting the dependence on $P$ )

$$
\mu_{K, Q}:=\left(d d^{c} V_{P, K, Q}^{*}\right)^{d} \text { and } \mu_{K}:=\left(d d^{c} V_{P, K}^{*}\right)^{d}
$$

for the Monge-Ampère measures of $V_{P, K, Q}^{*}$ and $V_{P, K}^{*}$ (the latter if $K$ is not pluripolar). Proposition 2.5 of [2] states that

$$
\operatorname{supp}\left(\mu_{K, Q}\right) \subset\left\{z \in K: V_{P, K, Q}^{*}(z) \geq Q(z)\right\}
$$

and $V_{P, K, Q}^{*}=Q$ q.e. on $\operatorname{supp}\left(\mu_{K, Q}\right)$, i.e., off of a pluripolar set.

\subsection{Energy}

We recall some results and definitions from [2]. For $u, v \in L_{P,+}\left(\mathbb{C}^{d}\right)$, we define the mutual energy

$$
\mathcal{E}(u, v):=\int_{\mathbb{C}^{d}}(u-v) \sum_{j=0}^{d}\left(d d^{c} u\right)^{j} \wedge\left(d d^{c} v\right)^{d-j} .
$$


For simplicity, when $v=H_{P}$, we denote the associated (normalized) energy functional by $E$ :

$$
E(u):=E_{H_{P}}(u)=\frac{1}{d+1} \sum_{j=0}^{d} \int_{\mathbb{C}^{d}}\left(u-H_{P}\right) d d^{c} u^{j} \wedge\left(d d^{c} H_{P}\right)^{d-j}
$$

(recall (2.7)).

For $u, u^{\prime}, v \in L_{P,+}\left(\mathbb{C}^{d}\right)$, and for $0 \leq t \leq 1$, we define

$$
f(t):=\mathcal{E}\left(u+t\left(u^{\prime}-u\right), v\right),
$$

From Proposition 3.1 in [2], $f^{\prime}(t)$ exists for $0 \leq t \leq 1$ and

$$
f^{\prime}(t)=(d+1) \int_{\mathbb{C}^{d}}\left(u^{\prime}-u\right)\left(d d^{c}\left(u+t\left(u^{\prime}-u\right)\right)\right)^{d}
$$

Hence, taking $v=H_{P}$, we have, for $F(t):=E\left(u+t\left(u^{\prime}-u\right)\right)$, that

$$
F^{\prime}(t)=\int_{\mathbb{C}^{d}}\left(u^{\prime}-u\right)\left(d d^{c}\left(u+t\left(u^{\prime}-u\right)\right)\right)^{d} .
$$

Thus $F^{\prime}(0)=\int_{\mathbb{C}^{d}}\left(u^{\prime}-u\right)\left(d d^{c} u\right)^{d}$ and we write

$$
<E^{\prime}(u), u^{\prime}-u>:=\int\left(u^{\prime}-u\right)\left(d d^{c} u\right)^{d} .
$$

We need some applications of a global domination principle. The following version, sufficient for our purposes, follows from [11], Corollary 3.10 (see also Corollary A.2 of [8]).

Proposition 3.1. Let $u \in L_{P}\left(\mathbb{C}^{d}\right)$ and $v \in \mathcal{E}_{P}\left(\mathbb{C}^{d}\right)$ with $u \leq v$ a.e. $\left(d d^{c} v\right)^{d}$. Then $u \leq v$ in $\mathbb{C}^{d}$.

This will be used to prove an approximation result, Proposition 3.3, which itself will be essential in the sequel. First we need a lemma.

Lemma 3.2. Assume that $\varphi \leq u, v \leq H_{P}$ are functions in $\mathcal{E}_{P}^{1}\left(\mathbb{C}^{d}\right)$. Then for all $t>0$,

$$
\int_{\left\{u \leq H_{P}-2 t\right\}}\left(H_{P}-u\right)\left(d d^{c} v\right)^{d} \leq 2^{d+1} \int_{\left\{\varphi \leq H_{P}-t\right\}}\left(H_{P}-\varphi\right)\left(d d^{c} \varphi\right)^{d} .
$$

In particular, the left hand side converges to 0 as $t \rightarrow+\infty$ uniformly in $u, v$. 
Proof. For $s>0$, we have the following inclusions of sets:

$$
\left(u \leq H_{P}-2 s\right) \subset\left(\varphi \leq \frac{v+H_{P}}{2}-s\right) \subset\left(\varphi \leq H_{P}-s\right) .
$$

We first note that the left hand side in the lemma is equal to

$$
\begin{aligned}
& \int_{\left\{u \leq H_{P}-2 t\right\}}\left(H_{P}-u\right)\left(d d^{c} v\right)^{d} \\
& \quad=2 t \int_{\left\{u \leq H_{P}-2 t\right\}}\left(d d^{c} v\right)^{d}+\int_{2 t}^{\infty}\left(\int_{\left\{u \leq H_{P}-s\right\}}\left(d d^{c} v\right)^{d}\right) d s .
\end{aligned}
$$

We claim that, for all $s>0$,

$$
\int_{\left\{u \leq H_{P}-2 s\right\}}\left(d d^{c} v\right)^{d} \leq 2^{d} \int_{\left\{\varphi \leq H_{P}-s\right\}}\left(d d^{c} \varphi\right)^{d} .
$$

Indeed, the comparison principle ([11, Corollary 3.6]) and the inclusions of sets above give

$$
\begin{aligned}
\int_{\left\{u \leq H_{P}-2 s\right\}}\left(d d^{c} v\right)^{d} & \leq \int_{\left\{\varphi \leq \frac{v+H_{P}}{2}-s\right\}}\left(d d^{c} v\right)^{d} \leq 2^{d} \int_{\left\{\varphi \leq \frac{v+H_{P}}{2}-s\right\}}\left(d d^{c} \frac{v+H_{P}}{2}\right)^{d} \\
& \leq 2^{d} \int_{\left\{\varphi \leq \frac{v+H_{P}}{2}-s\right\}}\left(d d^{c} \varphi\right)^{d} \leq 2^{d} \int_{\left\{\varphi \leq H_{P}-s\right\}}\left(d d^{c} \varphi\right)^{d} .
\end{aligned}
$$

The claim is proved. Using (3.3) and (3.2) we obtain

$$
\begin{aligned}
& \int_{\left\{u \leq H_{P}-2 t\right\}}\left(H_{P}-u\right)\left(d d^{c} v\right)^{d} \\
& \quad \leq 2^{d+1} t \int_{\left\{\varphi \leq H_{P}-t\right\}}\left(d d^{c} \varphi\right)^{d}+2^{d+1} \int_{t}^{+\infty}\left(\int_{\left\{\varphi \leq H_{P}-s\right\}}\left(d d^{c} \varphi\right)^{d}\right) d s \\
& =2^{d+1} \int_{\left\{\varphi \leq H_{P}-t\right\}}\left(H_{P}-\varphi\right)\left(d d^{c} \varphi\right)^{d} .
\end{aligned}
$$

Proposition 3.3. Let $u \in \mathcal{E}_{P}^{1}\left(\mathbb{C}^{d}\right)$ with $\left(d d^{c} u\right)^{d}=\mu$ having support in a nonpluripolar compact set $K$ so that $\int_{K} u d \mu>-\infty$ from Proposition 2.11. Let $\left\{Q_{j}\right\}$ be a sequence of continuous functions on $K$ decreasing to $u$ on $K$. Then $u_{j}:=V_{P, K, Q_{j}}^{*} \downarrow u$ on $\mathbb{C}^{d}$ and $\mu_{j}:=\left(d d^{c} u_{j}\right)^{d}$ is supported in $K$. In particular, $\mu_{j} \rightarrow \mu=\left(d d^{c} u\right)^{d}$ weak-*. Moreover,

$$
\lim _{j \rightarrow \infty} \int_{K} Q_{j} d \mu_{j}=\lim _{j \rightarrow \infty} \int_{K} Q_{j} d \mu=\int_{K} u d \mu>-\infty
$$


Proof. We can assume $\left\{Q_{j}\right\}$ are defined and decreasing to $u$ on the closure of a bounded open neighborhood $\Omega$ of $K$. By adding a negative constant we can assume that $Q_{1} \leq 0$ on $\Omega$. Since $\left\{Q_{j}\right\}$ is decreasing, so is the sequence $\left\{u_{j}\right\}$. Moreover, by [4, Proposition 5.1] $u_{j} \leq Q_{j}$ on $K \backslash E_{j}$ where $E_{j}$ is pluripolar. But $u$ is a competitor in the definition of $V_{P, K, Q_{j}}$ so that $u \leq u_{j}$ on $\mathbb{C}^{d}$. Thus $\tilde{u}:=\lim _{j \rightarrow \infty} u_{j} \geq u$ everywhere and $\tilde{u} \leq u$ on $K \backslash E$, where $E:=\cup_{j} E_{j}$ is a pluripolar set. Since $\left(d d^{c} u\right)^{d}$ puts no mass on pluripolar sets,

$$
\int_{\{u<\tilde{u}\}}\left(d d^{c} u\right)^{d} \leq \int_{E \cup\left(\mathbb{C}^{d} \backslash K\right)}\left(d d^{c} u\right)^{d}=0 .
$$

It thus follows from Proposition 3.1 that $\tilde{u} \leq u$, hence $\tilde{u}=u$ on $\mathbb{C}^{d}$.

The second equality in (3.4) follows from the monotone convergence theorem. It remains to prove that

$$
\lim _{j \rightarrow \infty} \int_{K}\left(-Q_{j}\right) d \mu_{j}=\int_{K}(-u) d \mu .
$$

For each $k$ fixed and $j \geq k$ we have

$$
\int_{K}\left(-Q_{j}\right) d \mu_{j} \geq \int_{K}\left(-Q_{k}\right) d \mu_{j}=\int_{\Omega}\left(-Q_{k}\right) d \mu_{j},
$$

hence $\liminf _{j \rightarrow \infty} \int_{K}\left(-Q_{j}\right) d \mu_{j} \geq \int_{K}\left(-Q_{k}\right) d \mu$ since $\Omega$ is open and $\mu_{j}, \mu$ are supported on $K$. Letting $k \rightarrow+\infty$ we arrive at

$$
\liminf _{j \rightarrow \infty} \int_{K}\left(-Q_{j}\right) d \mu_{j} \geq \int_{K}(-u) d \mu .
$$

It remains to prove that

$$
\limsup _{j \rightarrow \infty} \int_{K}\left(-Q_{j}\right) d \mu_{j} \leq \int_{K}(-u) d \mu .
$$

The sequence $\left\{u_{j}\right\}$ is not necessarily uniformly bounded below on $K$. However, using the facts that $Q_{j} \geq u$ and $H_{P}$ is continuous in $\mathbb{C}^{d}$, it suffices to prove that

$$
\limsup _{j \rightarrow \infty} \int_{K}\left(H_{P}-u\right)\left(d d^{c} u_{j}\right)^{d} \leq \int_{K}\left(H_{P}-u\right)\left(d d^{c} u\right)^{d} .
$$

To verify (3.5), we use Lemma 3.2.

By adding a negative constant we can assume that $u_{j} \leq H_{P}$. For a function $v$ and for $t>0$ we define $v^{t}:=\max \left(v, H_{P}-t\right)$. Note that for each $t$ the sequence $\left\{u_{j}^{t}\right\}$ is locally uniformly bounded below. Define

$$
a(t):=2^{d+1} \int_{\left\{u \leq H_{P}-t / 2\right\}}\left(H_{P}-u\right)\left(d d^{c} u\right)^{d} .
$$


Since $u \in \mathcal{E}_{P}^{1}\left(\mathbb{C}^{d}\right)$, from Proposition 2.11 we have $a(t) \rightarrow 0$ as $t \rightarrow+\infty$. By Lemma 3.2 we have

$$
\sup _{j \geq 1} \int_{\left\{u \leq H_{P}-t\right\}}\left(H_{P}-u\right)\left(d d^{c} u_{j}\right)^{d} \leq a(t)
$$

By the plurifine property of non-pluripolar Monge-Ampère measures [10, Proposition 1.4] and (3.6) we have

$$
\begin{aligned}
\int_{K}\left(H_{P}-u\right)\left(d d^{c} u_{j}\right)^{d} & \leq \int_{K \cap\left\{u>H_{P}-t\right\}}\left(H_{P}-u\right)\left(d d^{c} u_{j}\right)^{d}+a(t) \\
& =\int_{K \cap\left\{u>H_{P}-t\right\}}\left(H_{P}-u^{t}\right)\left(d d^{c} u_{j}^{t}\right)^{d}+a(t) \\
& \leq \int_{K}\left(H_{P}-u^{t}\right)\left(d d^{c} u_{j}^{t}\right)^{d}+a(t) .
\end{aligned}
$$

Since $H_{P}$ is bounded in $\Omega$, it follows from [16, Theorem 4.26] that the sequence of positive Radon measures $\left(H_{P}-u^{t}\right)\left(d d^{c} u_{j}^{t}\right)^{d}$ converges weakly on $\Omega$ to $\left(H_{P}-\right.$ $\left.u^{t}\right)\left(d d^{c} u^{t}\right)^{d}$. Since $K$ is compact it then follows that

$$
\limsup \int_{K}\left(H_{P}-u\right)\left(d d^{c} u_{j}\right)^{d} \leq \int_{K}\left(H_{P}-u^{t}\right)\left(d d^{c} u^{t}\right)^{d}+a(t) .
$$

We finally let $t \rightarrow+\infty$ to conclude the proof in the following manner:

$$
\begin{aligned}
\int_{K}\left(H_{P}-u^{t}\right)\left(d d^{c} u^{t}\right)^{d} & \leq \int_{K \cap\left\{u>H_{P}-t\right\}}\left(H_{P}-u^{t}\right)\left(d d^{c} u^{t}\right)^{d}+a(t) \\
& \leq \int_{K}\left(H_{P}-u\right)\left(d d^{c} u\right)^{d}+a(t),
\end{aligned}
$$

where in the first estimate we have used $\left\{u \leq H_{P}-t\right\}=\left\{u^{t} \leq H_{P}-t\right\}$ and Lemma 3.2 and in the last estimate we use again the plurifine property.

We now give an alternate description of the Legendre-type transform $E^{*}$ from (2.9) which will be related to the rate function in a large deviation principle. Given $K \subset \mathbb{C}^{d}$ compact, we let $\mathcal{M}_{P}(K)$ denote the space of positive measures on $K$ of total mass $\gamma_{d}$ and we let $C(K)$ denote the set of continuous, real-valued functions on $K$.

Proposition 3.4. Let $K$ be a nonpluripolar compact set and $\mu \in \mathcal{M}_{P}(K)$. Then

$$
E^{*}(\mu)=\sup _{v \in C(K)}\left[E\left(V_{P, K, v}^{*}\right)-\int_{K} v d \mu\right]
$$


Proof. We first treat the case when $E^{*}(\mu)=+\infty$. By Theorem 2.13 there exists $u \in \mathcal{E}_{P}^{1}\left(\mathbb{C}^{d}\right)$ such that $\int_{K} u d \mu=-\infty$. We take a decreasing sequence $Q_{j} \in C(K)$ such that $Q_{j} \downarrow u$ on $K$ and set $u_{j}:=V_{P, K, Q_{j}}^{*}$. Then $\left\{u_{j}\right\}$ are decreasing; since $u \in \mathcal{E}_{P}^{1}\left(\mathbb{C}^{d}\right)$ and $E$ is non-decreasing, $\left\{E\left(u_{j}\right)\right\}$ is uniformly bounded and we obtain

$$
E\left(V_{P, K, Q_{j}}^{*}\right)-\int_{K} Q_{j} d \mu \longrightarrow+\infty
$$

proving the proposition in this case.

Assume now that $E^{*}(\mu)<+\infty$. Theorem 2.13 ensures that $\int_{\mathbb{C}^{d}} u d \mu>-\infty$ for all $u \in \mathcal{E}_{P}^{1}\left(\mathbb{C}^{d}\right)$. By Lemma 2.15, $\mu$ puts no mass on pluripolar sets. From monotonicity of $E$ and the definition of $E^{*}$ in $(2.9)$ we have

$$
E^{*}(\mu) \geq \sup _{v \in C(K)}\left[E\left(V_{P, K, v}^{*}\right)-\int_{K} v d \mu\right] .
$$

Here we have used that

$$
V_{P, K, v}^{*} \leq v \text { q.e. on } K \text { for } v \in C(K)
$$

For the reverse inequality, fix $u \in \mathcal{E}_{P}^{1}\left(\mathbb{C}^{d}\right)$. Let $\left\{Q_{j}\right\}$ be a sequence of continuous functions on $K$ decreasing to $u$ on $K$ and set $u_{j}:=V_{P, K, Q_{j}}^{*}$. Given $\varepsilon>0$, we can choose $j$ sufficiently large so that, by monotone convergence,

$$
\int_{K} Q_{j} d \mu \leq \int_{K} u d \mu+\varepsilon
$$

and, by monotonicity of $E$,

$$
E\left(V_{P, K, Q_{j}}^{*}\right) \geq E(u)
$$

Hence

$$
E\left(V_{P, K, Q_{j}}^{*}\right)-\int_{K} Q_{j} d \mu \geq E(u)-\int_{K} u d \mu-\varepsilon
$$

so that

$$
\sup _{v \in C(K)}\left[E\left(V_{P, K, v}^{*}\right)-\int_{K} v d \mu\right] \geq E^{*}(\mu)
$$

and equality holds. 


\subsection{Transfinite diameter}

Let $d_{n}=d_{n}(P)$ denote the dimension of the vector space $\operatorname{Poly}(n P)$. We write

$$
\operatorname{Poly}(n P)=\operatorname{span}\left\{e_{1}, \ldots, e_{d_{n}}\right\}
$$

where $\left\{e_{j}(z):=z^{\alpha(j)}\right\}_{j=1, \ldots, d_{n}}$ are the standard basis monomials. Given $\zeta_{1}, \ldots, \zeta_{d_{n}} \in$ $\mathbb{C}^{d}$, let

$$
\begin{aligned}
\operatorname{VDM}\left(\zeta_{1}, \ldots, \zeta_{d_{n}}\right) & :=\operatorname{det}\left[e_{i}\left(\zeta_{j}\right)\right]_{i, j=1, \ldots, d_{n}} \\
& =\operatorname{det}\left[\begin{array}{cccc}
e_{1}\left(\zeta_{1}\right) & e_{1}\left(\zeta_{2}\right) & \ldots & e_{1}\left(\zeta_{d_{n}}\right) \\
\vdots & \vdots & \ddots & \vdots \\
e_{d_{n}}\left(\zeta_{1}\right) & e_{d_{n}}\left(\zeta_{2}\right) & \ldots & e_{d_{n}}\left(\zeta_{d_{n}}\right)
\end{array}\right]
\end{aligned}
$$

and for $K \subset \mathbb{C}^{d}$ compact let

$$
V_{n}=V_{n}(K):=\max _{\zeta_{1}, \ldots, \zeta_{d_{n}} \in K}\left|V D M\left(\zeta_{1}, \ldots, \zeta_{d_{n}}\right)\right| .
$$

It was shown in [2] that

$$
\delta(K):=\delta(K, P):=\lim _{n \rightarrow \infty} V_{n}^{1 / l_{n}}
$$

exists where

$$
l_{n}:=\sum_{j=1}^{d_{n}} \operatorname{deg}\left(e_{j}\right)=\sum_{j=1}^{d_{n}}|\alpha(j)|
$$

is the sum of the degrees of the basis monomials for Poly $(n P)$. We call $\delta(K)$ the $P$-transfinite diameter of $K$. More generally, for $w$ an admissible weight function on $K$ and $\zeta_{1}, \ldots, \zeta_{d_{n}} \in K$, let

$$
\begin{aligned}
V D M_{n}^{Q}\left(\zeta_{1}, \ldots, \zeta_{d_{n}}\right) & :=\operatorname{VDM}\left(\zeta_{1}, \ldots, \zeta_{d_{n}}\right) w\left(\zeta_{1}\right)^{n} \ldots w\left(\zeta_{d_{n}}\right)^{n} \\
& =\operatorname{det}\left[\begin{array}{cccc}
e_{1}\left(\zeta_{1}\right) & e_{1}\left(\zeta_{2}\right) & \ldots & e_{1}\left(\zeta_{d_{n}}\right) \\
\vdots & \vdots & \ddots & \vdots \\
e_{d_{n}}\left(\zeta_{1}\right) & e_{d_{n}}\left(\zeta_{2}\right) & \ldots & e_{d_{n}}\left(\zeta_{d_{n}}\right)
\end{array}\right] \cdot w\left(\zeta_{1}\right)^{n} \ldots w\left(\zeta_{d_{n}}\right)^{n}
\end{aligned}
$$

be a weighted Vandermonde determinant. Let

$$
W_{n}(K):=\max _{\zeta_{1}, \ldots, \zeta_{d_{n}} \in K}\left|V D M_{n}^{Q}\left(\zeta_{1}, \ldots, \zeta_{d_{n}}\right)\right| .
$$

An $n$-th weighted $P$-Fekete set for $K$ and $w$ is a set of $d_{n}$ points $\zeta_{1}, \ldots, \zeta_{d_{n}} \in K$ with the property that

$$
\left|V D M_{n}^{Q}\left(\zeta_{1}, \ldots, \zeta_{d_{n}}\right)\right|=W_{n}(K)
$$


The limit

$$
\delta^{Q}(K):=\delta^{Q}(K, P):=\lim _{n \rightarrow \infty} W_{n}(K)^{1 / l_{n}}
$$

exists and is called the weighted $P$-transfinite diameter. The following was proved in $[2]$.

Theorem 3.5. (Asymptotic Weighted $P$-Fekete Measures) Let $K \subset \mathbb{C}^{d}$ be compact with admissible weight $w$. For each $n$, take points $z_{1}^{(n)}, z_{2}^{(n)}, \ldots, z_{d_{n}}^{(n)} \in K$ for which

$$
\lim _{n \rightarrow \infty}\left[\left|V D M_{n}^{Q}\left(z_{1}^{(n)}, \ldots, z_{d_{n}}^{(n)}\right)\right|\right]^{\frac{1}{l_{n}}}=\delta^{Q}(K)
$$

(asymptotically weighted $P-$ Fekete arrays) and let $\mu_{n}:=\frac{1}{d_{n}} \sum_{j=1}^{d_{n}} \delta_{z_{j}^{(n)}}$. Then

$$
\mu_{n} \longrightarrow \frac{1}{\gamma_{d}} \mu_{K, Q} \text { weak-*. }
$$

Another ingredient we will use is a Rumely-type relation between transfinite diameter and energy of $V_{P, K, Q}^{*}$ from [2].

Theorem 3.6. Let $K \subset \mathbb{C}^{d}$ be compact and $w=e^{-Q}$ with $Q \in C(K)$. Then

$$
\log \delta^{Q}(K)=\frac{-1}{\gamma_{d} d A} \mathcal{E}\left(V_{P, K, Q}^{*}, H_{P}\right)=\frac{-(d+1)}{\gamma_{d} d A} E\left(V_{P, K, Q}^{*}\right)
$$

Here $A=A(P, d)$ was defined in [2]; we recall the definition. For $P=\Sigma$ so that $\operatorname{Poly}(n \Sigma)=\mathcal{P}_{n}$, we have

$$
d_{n}(\Sigma)=\left(\begin{array}{c}
d+n \\
d
\end{array}\right)=0\left(n^{d} / d !\right) \text { and } l_{n}(\Sigma)=\frac{d}{d+1} n d_{n}(\Sigma) .
$$

For a convex body $P \subset\left(\mathbb{R}^{+}\right)^{d}$, define $f_{n}(d)$ by writing

$$
l_{n}=f_{n}(d) \frac{n d}{d+1} d_{n}=f_{n}(d) \frac{l_{n}(\Sigma)}{d_{n}(\Sigma)} d_{n}
$$

Then the ratio $l_{n} / d_{n}$ divided by $l_{n}(\Sigma) / d_{n}(\Sigma)$ has a limit; i.e.,

$$
\lim _{n \rightarrow \infty} f_{n}(d)=: A=A(P, d)
$$




\subsection{Bernstein-Markov}

For $K \subset \mathbb{C}^{d}$ compact, $w=e^{-Q}$ an admissible weight function on $K$, and $\nu$ a finite measure on $K$, we say that the triple $(K, \nu, Q)$ satisfies a weighted BernsteinMarkov property if for all $p_{n} \in \mathcal{P}_{n}$,

$$
\left\|w^{n} p_{n}\right\|_{K} \leq M_{n}\left\|w^{n} p_{n}\right\|_{L^{2}(\nu)} \text { with } \limsup _{n \rightarrow \infty} M_{n}^{1 / n}=1 .
$$

Here, $\left\|w^{n} p_{n}\right\|_{K}:=\sup _{z \in K}\left|w(z)^{n} p_{n}(z)\right|$ and

$$
\left\|w^{n} p_{n}\right\|_{L^{2}(\nu)}^{2}:=\int_{K}\left|p_{n}(z)\right|^{2} w(z)^{2 n} d \nu(z) .
$$

Following [1], given $P \subset\left(\mathbb{R}^{+}\right)^{d}$ a convex body, we say that a finite measure $\nu$ with support in a compact set $K$ is a Bernstein-Markov measure for the triple $(P, K, Q)$ if (3.13) holds for all $p_{n} \in \operatorname{Poly}(n P)$.

For any $P$ there exists $A=A(P)>0$ with $\operatorname{Poly}(n P) \subset \mathcal{P}_{A n}$ for all $n$. Thus if $(K, \nu, Q)$ satisfies a weighted Bernstein-Markov property, then $\nu$ is a BernsteinMarkov measure for $(P, K, \widetilde{Q})$ where $\widetilde{Q}=A Q$. In particular, if $\nu$ is a strong Bernstein-Markov measure for $K$; i.e., if $\nu$ is a weighted Bernstein-Markov measure for any $Q \in C(K)$, then for any such $Q, \nu$ is a Bernstein-Markov measure for the triple $(P, K, Q)$. Strong Bernstein-Markov measures exist for any nonpluripolar compact set; cf., Corollary 3.8 of [9]. The paragraph following this corollary gives a sufficient mass-density type condition for a measure to be a strong Bernstein-Markov measure.

Given $P$, for $\nu$ a finite measure on $K$ and $Q \in \mathcal{A}(K)$, define

$$
Z_{n}:=Z_{n}(P, K, Q, \nu):=\int_{K} \ldots \int_{K}\left|V D M_{n}^{Q}\left(z_{1}, \ldots, z_{d_{n}}\right)\right|^{2} d \nu\left(z_{1}\right) \ldots d \nu\left(z_{d_{n}}\right) .
$$

The main consequence of using a Bernstein-Markov measure for $(P, K, Q)$ is the following:

Proposition 3.7. Let $K \subset \mathbb{C}^{d}$ be a compact set and let $Q \in \mathcal{A}(K)$. If $\nu$ is a Bernstein-Markov measure for $(P, K, Q)$ then

$$
\lim _{n \rightarrow \infty} Z_{n}^{\frac{1}{2 l_{n}}}=\delta^{Q}(K)
$$

Proof. That $\limsup _{n \rightarrow \infty} Z_{n}^{\frac{1}{2 l_{n}}} \leq \delta^{Q}(K)$ is clear. Observing from (3.7) and (3.9) that, fixing all variables but $z_{j}$,

$$
z_{j} \longrightarrow V D M_{n}^{Q}\left(z_{1}, \ldots, z_{j}, \ldots, z_{d_{n}}\right)=w\left(z_{j}\right)^{n} p_{n}\left(z_{j}\right)
$$

for some $p_{n} \in \operatorname{Poly}(n P)$, to show $\liminf _{n \rightarrow \infty} Z_{n}^{\frac{1}{2 l_{n}}} \geq \delta^{Q}(K)$ one starts with an $n$-th weighted $P$-Fekete set for $K$ and $w$ and repeatedly applies the weighted BernsteinMarkov property. 
Recall $\mathcal{M}_{P}(K)$ is the space of positive measures on $K$ with total mass $\gamma_{d}$. With the weak-* topology, this is a separable, complete metrizable space. A neighborhood basis of $\mu \in \mathcal{M}_{P}(K)$ can be given by sets

$$
\begin{gathered}
G(\mu, k, \varepsilon):=\left\{\sigma \in \mathcal{M}_{P}(K):\left|\int_{K}(\operatorname{Re} z)^{\alpha}(\operatorname{Im} z)^{\beta}(d \mu-d \sigma)\right|<\varepsilon\right. \\
\text { for } 0 \leq|\alpha|+|\beta| \leq k\}
\end{gathered}
$$

where $\operatorname{Re} z=\left(\operatorname{Re} z_{1}, \ldots, \operatorname{Re} z_{n}\right)$ and $\operatorname{Im} z=\left(\operatorname{Im} z_{1}, \ldots, \operatorname{Im} z_{n}\right)$.

Given $\nu$ as in Proposition 3.7, we define a probability measure $\operatorname{Prob}_{n}$ on $K^{d_{n}}$ via, for a Borel set $A \subset K^{d_{n}}$,

$$
\operatorname{Prob}_{n}(A):=\frac{1}{Z_{n}} \cdot \int_{A}\left|V D M_{n}^{Q}\left(z_{1}, \ldots, z_{d_{n}}\right)\right|^{2} \cdot d \nu\left(z_{1}\right) \ldots d \nu\left(z_{d_{n}}\right) .
$$

We immediately obtain the following:

Corollary 3.8. Let $\nu$ be a Bernstein-Markov measure for $(P, K, Q)$. Given $\eta>0$, define

$$
A_{n, \eta}:=\left\{\left(z_{1}, \ldots, z_{d_{n}}\right) \in K^{d_{n}}:\left|V D M_{n}^{Q}\left(z_{1}, \ldots, z_{d_{n}}\right)\right|^{2} \geq\left(\delta^{Q}(K)-\eta\right)^{2 l_{n}}\right\} .
$$

Then there exists $n^{*}=n^{*}(\eta)$ such that for all $n>n^{*}$,

$$
\operatorname{Prob}_{n}\left(K^{d_{n}} \backslash A_{n, \eta}\right) \leq\left(1-\frac{\eta}{2 \delta^{Q}(K)}\right)^{2 l_{n}}
$$

Remark 3.9. Corollary 3.8 was proved in [9], Corollary 3.2, for $\nu$ a probability measure but an obvious modification works for $\nu(K)<\infty$.

Using (3.17), we get an induced probability measure $\mathbf{P}$ on the infinite product space of arrays $\chi:=\left\{X=\left\{x_{j}^{(n)}\right\}_{n=1,2, \ldots ; j=1, \ldots, d_{n}}: x_{j}^{(n)} \in K\right\}$ :

$$
(\chi, \mathbf{P}):=\prod_{n=1}^{\infty}\left(K^{d_{n}}, \operatorname{Prob}_{n}\right) .
$$

Corollary 3.10. Let $\nu$ be a Bernstein-Markov measure for $(P, K, Q)$. For P-a.e. array $X=\left\{x_{j}^{(n)}\right\} \in \chi$,

$$
\nu_{n}:=\frac{1}{d_{n}} \sum_{j=1}^{d_{n}} \delta_{x_{j}^{(n)}} \longrightarrow \frac{1}{\gamma_{d}} \mu_{K, Q} \text { weak- * }
$$


Proof. From Theorem 3.5 it suffices to verify for $\mathbf{P}$-a.e. array $X=\left\{x_{j}^{(n)}\right\}$

$$
\liminf _{n \rightarrow \infty}\left(\left|V D M_{n}^{Q}\left(x_{1}^{(n)}, \ldots, x_{d_{n}}^{(n)}\right)\right|\right)^{\frac{1}{l_{n}}}=\delta^{Q}(K) .
$$

Given $\eta>0$, the condition that for a given array $X=\left\{x_{j}^{(n)}\right\}$ we have

$$
\liminf _{n \rightarrow \infty}\left(\left|V D M_{n}^{Q}\left(x_{1}^{(n)}, \ldots, x_{d_{n}}^{(n)}\right)\right|\right)^{\frac{1}{l_{n}}} \leq \delta^{Q}(K)-\eta
$$

means that $\left(x_{1}^{(n)}, \ldots, x_{d_{n}}^{(n)}\right) \in K^{d_{n}} \backslash A_{n, \eta}$ for infinitely many $n$. Setting

$$
E_{n}:=\left\{X \in \chi:\left(x_{1}^{(n)}, \ldots, x_{d_{n}}^{(n)}\right) \in K^{d_{n}} \backslash A_{n, \eta}\right\},
$$

we have

$$
\mathbf{P}\left(E_{n}\right) \leq \operatorname{Prob}_{n}\left(K^{d_{n}} \backslash A_{n, \eta}\right) \leq\left(1-\frac{\eta}{2 \delta^{Q}(K)}\right)^{2 l_{n}}
$$

and $\sum_{n=1}^{\infty} \mathbf{P}\left(E_{n}\right)<+\infty$. By the Borel-Cantelli lemma,

$$
\mathbf{P}\left(\limsup _{n \rightarrow \infty} E_{n}\right)=\mathbf{P}\left(\bigcap_{n=1}^{\infty} \bigcup_{k \geq n}^{\infty} E_{k}\right)=0 .
$$

Thus, with probability one, only finitely many $E_{n}$ occur, and (3.19) follows.

The main goal in the rest of the paper is to verify a stronger probabilistic result - a large deviation principle - and to explain this result in $P$-pluripotentialtheoretic terms.

\section{Relation between $E^{*}$ and $J, J^{Q}$ functionals}

We define some functionals on $\mathcal{M}_{P}(K)$ using $L^{2}$-type notions which act as a replacement for an energy functional on measures. Then we show these functionals $\bar{J}(\mu)$ and $\underline{J}(\mu)$ defined using a "lim sup" and a "liminf" coincide (see Definitions 4.1 and 4.2 ); this is the essence of our first proof of the large deviation principle, Theorem 5.1. Using Proposition 3.4, we relate this functional with $E^{*}$ from (2.9).

Fix a nonpluripolar compact set $K$ and a strong Bernstein-Markov measure $\nu$ on $K$. For simplicity, we normalize so that $\nu$ is a probability measure. Recall then for any $Q \in C(K), \nu$ is a Bernstein-Markov measure for the triple $(P, K, Q)$. Given $G \subset \mathcal{M}_{P}(K)$ open, for each $s=1,2, \ldots$ we set

$$
\widetilde{G}_{s}:=\left\{\mathbf{a}=\left(a_{1}, \ldots, a_{s}\right) \in K^{s}: \frac{\gamma_{d}}{s} \sum_{j=1}^{s} \delta_{a_{j}} \in G\right\} .
$$


Define, for $n=1,2, \ldots$,

$$
J_{n}(G):=\left[\int_{\widetilde{G}_{d_{n}}}\left|V D M_{n}(\mathbf{a})\right|^{2} d \nu(\mathbf{a})\right]^{1 / 2 l_{n}} .
$$

Definition 4.1. For $\mu \in \mathcal{M}_{P}(K)$ we define

$$
\begin{aligned}
& \bar{J}(\mu):=\inf _{G \ni \mu} \bar{J}(G) \text { where } \bar{J}(G):=\limsup _{n \rightarrow \infty} J_{n}(G) ; \\
& \underline{J}(\mu):=\inf _{G \ni \mu} \underline{J}(G) \text { where } \underline{J}(G):=\liminf _{n \rightarrow \infty} J_{n}(G) .
\end{aligned}
$$

The infima are taken over all neighborhoods $G$ of the measure $\mu$ in $\mathcal{M}_{P}(K)$. A priori, $\bar{J}, \underline{J}$ depend on $\nu$. These functionals are nonnegative but can take the value zero. Intuitively, we are taking a "limit" of $L^{2}(\nu)$ averages of discrete, equally weighted approximants $\frac{\gamma_{d}}{s} \sum_{j=1}^{s} \delta_{a_{j}}$ of $\mu$. An " $L^{\infty}$ " version of $\bar{J}, \underline{J}$ was introduced in [8] where $J_{n}(G)$ is replaced by

$$
W_{n}(G):=\sup _{\mathbf{a} \in \widetilde{G}_{d_{n}}}\left|V D M_{n}(\mathbf{a})\right|^{1 / l_{n}} \geq J_{n}(G) .
$$

The weighted versions of these functionals are defined for $Q \in \mathcal{A}(K)$ using

$$
J_{n}^{Q}(G):=\left[\int_{\widetilde{G}_{d_{n}}}\left|V D M_{n}^{Q}(\mathbf{a})\right|^{2} d \nu(\mathbf{a})\right]^{1 / 2 l_{n}} .
$$

Definition 4.2. For $\mu \in \mathcal{M}_{P}(K)$ we define

$$
\begin{aligned}
& \bar{J}^{Q}(\mu):=\inf _{G \ni \mu} \bar{J}^{Q}(G) \text { where } \bar{J}^{Q}(G):=\limsup _{n \rightarrow \infty} J_{n}^{Q}(G) ; \\
& \underline{J}^{Q}(\mu):=\inf _{G \ni \mu} \underline{J}^{Q}(G) \text { where } \underline{J}^{Q}(G):=\liminf _{n \rightarrow \infty} J_{n}^{Q}(G) .
\end{aligned}
$$

The uppersemicontinuity of $\bar{J}, \bar{J}^{Q}, \underline{J}$ and $\underline{J}^{Q}$ on $\mathcal{M}_{P}(K)$ (with the weak-* topology) follows as in Lemma 3.1 of [8]. Set

$$
b_{d}=b_{d}(P):=\frac{d+1}{A d \gamma_{d}} .
$$

Proposition 4.3. Fix $Q \in C(K)$. Then

(1) $\bar{J}^{Q}(\mu) \leq \delta^{Q}(K)$;

(2) $\bar{J}(\mu)=\bar{J}^{Q}(\mu) \cdot\left(e^{\int_{K} Q d \mu}\right)^{b_{d}}$;

(3) $\log \bar{J}(\mu) \leq \inf _{v \in C(K)}\left[\log \delta^{v}(K)+b_{d} \int_{K} v d \mu\right]$;

(4) $\log \bar{J}^{Q}(\mu) \leq \inf _{v \in C(K)}\left[\log \delta^{v}(K)+b_{d} \int_{K} v d \mu\right]-b_{d} \int_{K} Q d \mu$.

Properties (1)-(4) also hold for the functionals $\underline{J}, \underline{J}^{Q}$. 
Proof. Property (1) follows from

$$
J_{n}^{Q}(G) \leq \sup _{\mathbf{a} \in \widetilde{G}_{d_{n}}}\left|V D M_{n}^{Q}(\mathbf{a})\right|^{1 / l_{n}} \leq \sup _{\mathbf{a} \in K^{d_{n}}}\left|V D M_{n}^{Q}(\mathbf{a})\right|^{1 / l_{n}} .
$$

The proofs of Corollary 3.4, Proposition 3.5 and Proposition 3.6 of [8] work mutatis mutandis to verify (2), (3) and (4). The relevant estimation, replacing the corresponding one which is two lines above equation (3.2) in [8], is, given $\varepsilon>0$, for $\mathbf{a} \in \widetilde{G}_{d_{n}}$,

$$
\begin{aligned}
\left|V D M_{n}^{Q}(\mathbf{a})\right| e^{\frac{n d_{n}}{\gamma_{d}}\left(-\varepsilon-\int_{K} Q d \mu\right)} & \leq\left|V D M_{n}(\mathbf{a})\right| \\
& \leq\left|V D M_{n}^{Q}(\mathbf{a})\right| e^{\frac{n d_{n}}{\gamma_{d}}\left(\varepsilon+\int_{K} Q d \mu\right)} .
\end{aligned}
$$

To see this, we first recall that

$$
\left|V D M_{n}(\mathbf{a})\right|=\left|V D M_{n}^{Q}(\mathbf{a})\right| e^{n \sum_{j=1}^{d_{n}} Q\left(a_{j}\right)} .
$$

For $\mu \in \mathcal{M}_{P}(K), Q \in C(K), \varepsilon>0$, there exists a neighborhood $G$ of $\mu$ in $\mathcal{M}_{P}(K)$ with

$$
-\varepsilon<\int_{K} Q d \mu-\frac{\gamma_{d}}{d_{n}} \sum_{j=1}^{d_{n}} Q\left(a_{j}\right)<\varepsilon
$$

for $\mathbf{a} \in \widetilde{G}_{d_{n}}$. Plugging this double inequality into the previous equality we get (4.4). Moreover, from (3.12),

$$
\lim _{n \rightarrow \infty} \frac{n d_{n}}{l_{n}}=\frac{d+1}{A d}=b_{d} \gamma_{d}
$$

so that $\frac{n d_{n}}{\gamma_{d}} \asymp l_{n} b_{d}$ as $n \rightarrow \infty$. Taking $l_{n}-$ th roots in (4.4) accounts for the factor of $b_{d}$ in $(2),(3)$ and $(4)$.

Remark 4.4. The corresponding $\underline{W}, \underline{W}^{Q}, \bar{W}, \bar{W}^{Q}$ functionals, defined using (4.2), clearly dominate their " $J$ " counterparts; e.g., $\bar{W}^{Q} \geq \bar{J}^{Q}$.

Note that formula (3.11) can be rewritten:

$$
\log \delta^{Q}(K)=-b_{d} E\left(V_{P, K, Q}^{*}\right) .
$$

Thus the upper bound in Proposition 4.3 (3) becomes

$$
\log \bar{J}(\mu) \leq-b_{d} \sup _{v \in C(K)}\left[E\left(V_{P, K, v}^{*}\right)-\int_{K} v d \mu\right]=-b_{d} E^{*}(\mu) .
$$

For the rest of section 4 and section 5, we will always assume $Q \in C(K)$. Theorem 4.5 shows that the inequalities in (3) and (4) are equalities, and that the $\bar{J}, \bar{J}^{Q}$ functionals coincide with their $\underline{J}, \underline{J}^{Q}$ counterparts. The key step in the proof of Theorem 4.5 is to verify this for $\bar{J}^{v}\left(\mu_{K, v}\right)$ and $\underline{J}^{v}\left(\mu_{K, v}\right)$. 
Theorem 4.5. Let $K \subset \mathbb{C}^{d}$ be a nonpluripolar compact set and let $\nu$ satisfy a strong Bernstein-Markov property. Fix $Q \in C(K)$. Then for any $\mu \in \mathcal{M}_{P}(K)$,

$$
\log \bar{J}(\mu)=\log \underline{J}(\mu)=\inf _{v \in C(K)}\left[\log \delta^{v}(K)+b_{d} \int_{K} v d \mu\right]
$$

and

$$
\log \bar{J}^{Q}(\mu)=\log \underline{J}^{Q}(\mu)=\inf _{v \in C(K)}\left[\log \delta^{v}(K)+b_{d} \int_{K} v d \mu\right]-b_{d} \int_{K} Q d \mu .
$$

Proof. It suffices to prove (4.8) since (4.9) follows from (2) of Proposition 4.3. We have the upper bound

$$
\log \bar{J}(\mu) \leq \inf _{v \in C(K)}\left[\log \delta^{v}(K)+b_{d} \int_{K} v d \mu\right]
$$

from (3); for the lower bound, we consider different cases.

Case I: $\mu=\mu_{K, v}$ for some $v \in C(K)$.

We verify that

$$
\log \bar{J}\left(\mu_{K, v}\right)=\log \underline{J}\left(\mu_{K, v}\right)=\log \delta^{v}(K)+b_{d} \int_{K} v d \mu_{K, v}
$$

which proves (4.8) in this case.

To prove (4.10), we use the definition of $\underline{J}\left(\mu_{K, v}\right)$ and Corollary 3.8. Fix a neighborhood $G$ of $\mu_{K, v}$. For $\eta>0$, define $A_{n, \eta}$ as in (3.18) with $Q=v$. Set

$$
\eta_{n}:=\max \left(\delta^{v}(K)-\frac{n Z_{n}^{1 / 2 l_{n}}}{n+1}, \frac{Z_{n}^{1 / 2 l_{n}}}{n+1}\right) .
$$

By Proposition 3.7, $\eta_{n} \rightarrow 0$. We claim that we have the inclusion

$$
A_{n, \eta_{n}} \subset \widetilde{G}_{d_{n}} \text { for all } n \text { large enough. }
$$

We prove (4.12) by contradiction: if false, there is a sequence $\left\{n_{j}\right\}$ with $n_{j} \uparrow \infty$ and $x^{j}=\left(x_{1}^{j}, \ldots, x_{d_{n_{j}}}^{j}\right) \in A_{n_{j}, \eta_{n_{j}}} \backslash \widetilde{G}_{d_{n_{j}}}$. However $\mu_{j}:=\frac{\gamma_{d}}{d_{n_{j}}} \sum_{i=1}^{d_{n_{j}}} \delta_{x_{i}^{j}} \notin G$ for $j$ sufficiently large contradicts Theorem 3.5 since $x^{j} \in A_{n_{j}, \eta_{j}}$ and $\eta_{j} \downarrow 0$ imply $\mu_{j} \rightarrow \mu_{K, v}$ weak-*.

Next, a direct computation using (4.11) shows that, for all $n$ large enough,

$$
\operatorname{Prob}_{n}\left(K^{d_{n}} \backslash A_{n, \eta_{n}}\right) \leq \frac{\left(\delta^{v}(K)-\eta_{n}\right)^{2 l_{n}}}{Z_{n}} \leq\left(\frac{n}{n+1}\right)^{2 l_{n}} \leq \frac{n}{n+1}
$$


(recall $\nu$ is a probability measure). Hence

$$
\begin{aligned}
& \frac{1}{Z_{n}} \int_{\widetilde{G}_{d_{n}}}\left|V D M_{n}^{v}\left(z_{1}, \ldots, z_{d_{n}}\right)\right|^{2} \cdot d \nu\left(z_{1}\right) \ldots d \nu\left(z_{d_{n}}\right) \\
& \quad \geq \frac{1}{Z_{n}} \int_{A_{n, \eta_{n}}}\left|V D M_{n}^{v}\left(z_{1}, \ldots, z_{d_{n}}\right)\right|^{2} \cdot d \nu\left(z_{1}\right) \ldots d \nu\left(z_{d_{n}}\right) \\
& \quad \geq \frac{1}{n+1} .
\end{aligned}
$$

Since $P \subset r \Sigma$ and $\Sigma \subset k P$ for some $k \in \mathbb{Z}^{+}, l_{n}=0\left(n^{d+1}\right)$ and we have $\frac{1}{2 l_{n}} \log (n+1) \rightarrow 0$. Since $\nu$ satisfies a strong Bernstein-Markov property and $v \in C(K)$, using Proposition 3.7 and the above estimate we conclude that

$$
\begin{aligned}
& \liminf _{n \rightarrow \infty} \frac{1}{2 l_{n}} \log \int_{\widetilde{G}_{d_{n}}}\left|V D M_{n}^{v}\left(z_{1}, \ldots, z_{d_{n}}\right)\right|^{2} d \nu\left(z_{1}\right) \ldots d \nu\left(z_{d_{n}}\right) \\
& \quad \geq \log \delta^{v}(K) .
\end{aligned}
$$

Taking the infimum over all neighborhoods $G$ of $\mu_{K, v}$ we obtain

$$
\log \underline{J}^{v}\left(\mu_{K, v}\right) \geq \log \delta^{v}(K) .
$$

From (1) Proposition 4.3, $\log \bar{J}^{v}\left(\mu_{K, v}\right) \leq \log \delta^{v}(K)$; thus we have

$$
\log \underline{J}^{v}\left(\mu_{K, v}\right)=\log \bar{J}^{v}\left(\mu_{K, v}\right)=\log \delta^{v}(K) .
$$

Using (2) of Proposition 4.3 with $\mu=\mu_{K, v}$ we obtain (4.10).

Case II: $\mu \in \mathcal{M}_{P}(K)$ with the property that $E^{*}(\mu)<\infty$.

From Theorem 2.13 and Proposition 2.11 there exists $u \in L_{P}\left(\mathbb{C}^{d}\right)$ - indeed, $u \in \mathcal{E}_{P}^{1}\left(\mathbb{C}^{d}\right)$ - with $\mu=\left(d d^{c} u\right)^{d}$ and $\int_{K} u d \mu>-\infty$. However, since $u$ is only usc on $K, \mu$ is not necessarily of the form $\mu_{K, v}$ for some $v \in C(K)$. Taking a sequence of continuous functions $\left\{Q_{j}\right\} \subset C(K)$ with $Q_{j} \downarrow u$ on $K$, by Proposition 3.3 the weighted extremal functions $V_{P, K, Q_{j}}^{*}$ decrease to $u$ on $\mathbb{C}^{d}$;

$$
\mu_{j}:=\left(d d^{c} V_{P, K, Q_{j}}^{*}\right)^{d} \longrightarrow \mu=\left(d d^{c} u\right)^{d} \text { weak-*; }
$$

and

$$
\lim _{j \rightarrow \infty} \int_{K} Q_{j} d \mu_{j}=\lim _{j \rightarrow \infty} \int_{K} Q_{j} d \mu=\int_{K} u d \mu .
$$

From the previous case we have

$$
\log \bar{J}\left(\mu_{j}\right)=\log \underline{J}\left(\mu_{j}\right)=\log \delta^{Q_{j}}(K)+b_{d} \int_{K} Q_{j} d \mu_{j} .
$$


Using uppersemicontinuity of the functional $\mu \rightarrow \underline{J}(\mu)$,

$$
\limsup _{j \rightarrow \infty} \bar{J}\left(\mu_{j}\right)=\limsup _{j \rightarrow \infty} \underline{J}\left(\mu_{j}\right) \leq \underline{J}(\mu) .
$$

Since $Q_{j} \downarrow u$ on $K$,

$$
\limsup _{j \rightarrow \infty} \log \delta^{Q_{j}}(K)=\lim _{j \rightarrow \infty} \log \delta^{Q_{j}}(K)
$$

Therefore

$$
M:=\lim _{j \rightarrow \infty} \log \underline{J}\left(\mu_{j}\right)=\lim _{j \rightarrow \infty}\left(\log \delta^{Q_{j}}(K)+b_{d} \int_{K} Q_{j} d \mu_{j}\right)
$$

exists and is less than or equal to $\log \underline{J}(\mu)$. We want to show that

$$
\inf _{v}\left[\log \delta^{v}(K)+b_{d} \int_{K} v d \mu\right] \leq M .
$$

Given $\varepsilon>0$, by $(4.15)$ for $j \geq j_{0}(\varepsilon)$,

$$
\int_{K} Q_{j} d \mu_{j} \geq \int_{K} Q_{j} d \mu-\varepsilon \text { and } \log \underline{J}\left(\mu_{j}\right)<M+\varepsilon .
$$

Hence for such $j$,

$$
\begin{aligned}
\inf _{v}\left[\log \delta^{v}(K)+b_{d} \int_{K} v d \mu\right] & \leq \log \delta^{Q_{j}}(K)+b_{d} \int_{K} Q_{j} d \mu \\
& \leq \log \delta^{Q_{j}}(K)+b_{d} \int_{K} Q_{j} d \mu_{j}+b_{d} \varepsilon \\
& =\log \underline{J}\left(\mu_{j}\right)+b_{d} \varepsilon<M+\left(b_{d}+1\right) \varepsilon,
\end{aligned}
$$

yielding (4.17). This finishes the proof in Case II.

Case III: $\mu \in \mathcal{M}(K)$ with the property that $E^{*}(\mu)=+\infty$.

It follows from Proposition 3.4 and Theorem 3.6 that the right-hand side of (4.8) is $-\infty$, finishing the proof.

Remark 4.6. From now on, we simply use the notation $J, J^{Q}$ without the overline or underline. Using Proposition 3.4 and Theorem 3.6, we have

$$
\begin{aligned}
\log J(\mu) & =\inf _{Q \in C(K)}\left[\log \delta^{Q}(K)+b_{d} \int_{K} Q d \mu\right] \\
& =-\sup _{Q \in C(K)}\left[-\log \delta^{Q}(K)-b_{d} \int_{K} Q d \mu\right] \\
& =-\sup _{Q \in C(K)}\left[b_{d} E\left(V_{P, K, Q}^{*}\right)-b_{d} \int_{K} Q d \mu\right]=-b_{d} \sup _{Q \in C(K)}\left[E\left(V_{P, K, Q}^{*}\right)-\int_{K} Q d \mu\right]
\end{aligned}
$$


(recall (4.6)) which one can compare with

$$
E^{*}(\mu)=\sup _{Q \in C(K)}\left[E\left(V_{P, K, Q}^{*}\right)-\int_{K} Q d \mu\right]
$$

from Proposition 3.4 to conclude

$$
\log J(\mu)=-b_{d} E^{*}(\mu) .
$$

In particular, $J, J^{Q}$ are independent of the choice of strong Bernstein-Markov measure for $K$.

Following the idea in Proposition 4.3 of [9], we observe the following:

Proposition 4.7. Let $K \subset \mathbb{C}^{d}$ be a nonpluripolar compact set and let $\nu$ satisfy a strong Bernstein-Markov property. Fix $Q \in C(K)$. The measure $\mu_{K, Q}$ is the unique maximizer of the functional $\mu \rightarrow J^{Q}(\mu)$ over $\mu \in \mathcal{M}_{P}(K)$; i.e.,

$$
J^{Q}\left(\mu_{K, Q}\right)=\delta^{Q}(K)\left(\text { and } J\left(\mu_{K}\right)=\delta(K)\right) .
$$

Proof. The fact that $\mu_{K, Q}$ maximizes $J^{Q}$ (and $\mu_{K}$ maximizes $J$ ) follows from (4.10), (4.14) and Proposition 4.3.

Assume now that $\mu \in \mathcal{M}_{P}(K)$ maximizes $J^{Q}$. From Remark 4.4 and the definitions of the functionals, for any neighborhood $G \subset \mathcal{M}_{P}(K)$ of $\mu$,

$$
\bar{J}^{Q}(\mu) \leq \bar{W}^{Q}(\mu) \leq \sup \left\{\limsup _{n \rightarrow \infty}\left|V D M_{n}^{Q}\left(\mathbf{a}^{(n)}\right)\right|^{1 / l_{n}}\right\} \leq \delta^{Q}(K)
$$

where the supremum is taken over all arrays $\left\{\mathbf{a}^{(n)}\right\}_{n=1,2, \ldots}$ of $d_{n}$-tuples $\mathbf{a}^{(n)}$ in $K$ whose normalized counting measures $\mu_{n}:=\frac{1}{d_{n}} \sum_{j=1}^{d_{n}} \delta_{a_{j}^{(n)}}$ lie in $G$. Since $\bar{J}^{Q}(\mu)=$ $\delta^{Q}(K)$ there is an asymptotic weighted Fekete array $\left\{\mathbf{a}^{(n)}\right\}$ as in (3.10). Theorem 3.5 yields that $\mu_{n}:=\frac{1}{d_{n}} \sum_{j=1}^{d_{n}} \delta_{a_{j}^{(n)}}$ converges weak-* to $\mu_{K, Q}$, hence $\mu_{K, Q} \in \bar{G}$. Since this is true for each neighborhood $G \subset \mathcal{M}_{P}(K)$ of $\mu$, we must have $\mu=\mu_{K, Q}$.

\section{Large deviation}

As in the previous section, we fix $K \subset \mathbb{C}^{d}$ a nonpluripolar compact set; $Q \in$ $C(K)$; and a measure $\nu$ on $K$ satisfying a strong Bernstein-Markov property. For $x_{1}, \ldots, x_{d_{n}} \in K$, we get a discrete measure $\frac{\gamma_{d}}{d_{n}} \sum_{j=1}^{d_{n}} \delta_{x_{j}} \in \mathcal{M}_{P}(K)$. Define $j_{n}: K^{d_{n}} \rightarrow$ $\mathcal{M}_{P}(K)$ via

$$
j_{n}\left(x_{1}, \ldots, x_{d_{n}}\right):=\frac{\gamma_{d}}{d_{n}} \sum_{j=1}^{d_{n}} \delta_{x_{j}} .
$$


From $(3.17), \sigma_{n}:=\left(j_{n}\right)_{*}\left(\operatorname{Prob}_{n}\right)$ is a probability measure on $\mathcal{M}_{P}(K)$ : for a Borel set $B \subset \mathcal{M}_{P}(K)$,

$$
\sigma_{n}(B)=\frac{1}{Z_{n}} \int_{\widetilde{B}_{d_{n}}}\left|V D M_{n}^{Q}\left(x_{1}, \ldots, x_{d_{n}}\right)\right|^{2} d \nu\left(x_{1}\right) \ldots d \nu\left(x_{d_{n}}\right)
$$

where $\widetilde{B}_{d_{n}}:=\left\{\mathbf{a}=\left(a_{1}, \ldots, a_{d_{n}}\right) \in K^{d_{n}}: \frac{\gamma_{d}}{d_{n}} \sum_{j=1}^{d_{n}} \delta_{a_{j}} \in B\right\}($ recall $(4.1))$. Here, $Z_{n}:=$ $Z_{n}(P, K, Q, \nu)$. Note that

$$
\sigma_{n}(B)^{1 / 2 l_{n}}=\frac{1}{Z_{n}^{1 / 2 l_{n}}} \cdot J_{n}^{Q}(B) .
$$

For future use, suppose we have a function $F: \mathbb{R} \rightarrow \mathbb{R}$ and a function $v \in C(K)$. We write, for $\mu \in \mathcal{M}_{P}(K)$,

$$
<v, \mu>:=\int_{K} v d \mu
$$

and then

$$
\begin{aligned}
& \int_{\mathcal{M}_{P}(K)} F(<v, \mu>) d \sigma_{n}(\mu) \\
& \quad:=\frac{1}{Z_{n}} \int_{K} \ldots \int_{K}\left|V D M_{n}^{Q}\left(x_{1}, \ldots, x_{d_{n}}\right)\right|^{2} F\left(\frac{\gamma_{d}}{d_{n}} \sum_{j=1}^{d_{n}} v\left(x_{j}\right)\right) d \nu\left(x_{1}\right) \ldots d \nu\left(x_{d_{n}}\right) .
\end{aligned}
$$

With this notation, we offer two proofs of our LDP, Theorem 5.1. We state the result; define LDP in Definition 5.2; and then proceed with the proofs. This closely follows the exposition in section 5 of [9].

Theorem 5.1. The sequence $\left\{\sigma_{n}=\left(j_{n}\right)_{*}\left(\right.\right.$ Prob $\left.\left._{n}\right)\right\}$ of probability measures on $\mathcal{M}_{P}(K)$ satisfies a large deviation principle with speed $2 l_{n}$ and good rate function $\mathcal{I}:=\mathcal{I}_{K, Q}$ where, for $\mu \in \mathcal{M}_{P}(K)$,

$$
\mathcal{I}(\mu):=\log J^{Q}\left(\mu_{K, Q}\right)-\log J^{Q}(\mu) .
$$

This means that $\mathcal{I}: \mathcal{M}_{P}(K) \rightarrow[0, \infty]$ is a lowersemicontinuous mapping such that the sublevel sets $\left\{\mu \in \mathcal{M}_{P}(K): \mathcal{I}(\mu) \leq \alpha\right\}$ are compact in the weak-* topology on $\mathcal{M}_{P}(K)$ for all $\alpha \geq 0$ ( $\mathcal{I}$ is "good") satisfying (5.4) and (5.5):

Definition 5.2. The sequence $\left\{\mu_{n}\right\}$ of probability measures on $\mathcal{M}_{P}(K)$ satisfies a large deviation principle (LDP) with good rate function $\mathcal{I}$ and speed $2 l_{n}$ if for all measurable sets $\Gamma \subset \mathcal{M}_{P}(K)$,

$$
-\inf _{\mu \in \Gamma^{0}} \mathcal{I}(\mu) \leq \liminf _{n \rightarrow \infty} \frac{1}{2 l_{n}} \log \mu_{n}(\Gamma) \text { and }
$$




$$
\limsup _{n \rightarrow \infty} \frac{1}{2 l_{n}} \log \mu_{n}(\Gamma) \leq-\inf _{\mu \in \bar{\Gamma}} \mathcal{I}(\mu) .
$$

In the setting of $\mathcal{M}_{P}(K)$, to prove a LDP it suffices to work with a base for the weak-* topology. The following is a special case of a basic general existence result for a LDP given in Theorem 4.1.11 in [13].

Proposition 5.3. Let $\left\{\sigma_{\varepsilon}\right\}$ be a family of probability measures on $\mathcal{M}_{P}(K)$. Let $\mathcal{B}$ be a base for the topology of $\mathcal{M}_{P}(K)$. For $\mu \in \mathcal{M}_{P}(K)$ let

$$
\mathcal{I}(\mu):=-\inf _{\{G \in \mathcal{B}: \mu \in G\}}\left(\liminf _{\varepsilon \rightarrow 0} \varepsilon \log \sigma_{\varepsilon}(G)\right) .
$$

Suppose for all $\mu \in \mathcal{M}_{P}(K)$,

$$
\mathcal{I}(\mu)=-\inf _{\{G \in \mathcal{B}: \mu \in G\}}\left(\limsup _{\varepsilon \rightarrow 0} \varepsilon \log \sigma_{\varepsilon}(G)\right) .
$$

Then $\left\{\sigma_{\varepsilon}\right\}$ satisfies a LDP with rate function $\mathcal{I}(\mu)$ and speed $1 / \varepsilon$.

There is a converse to Proposition 5.3, Theorem 4.1.18 in [13]. For $\mathcal{M}_{P}(K)$, it reads as follows:

Proposition 5.4. Let $\left\{\sigma_{\varepsilon}\right\}$ be a family of probability measures on $\mathcal{M}_{P}(K)$. Suppose that $\left\{\sigma_{\varepsilon}\right\}$ satisfies a LDP with rate function $\mathcal{I}(\mu)$ and speed $1 / \varepsilon$. Then for any base $\mathcal{B}$ for the topology of $\mathcal{M}_{P}(K)$ and any $\mu \in \mathcal{M}_{P}(K)$

$$
\begin{aligned}
\mathcal{I}(\mu) & :=-\inf _{\{G \in \mathcal{B}: \mu \in G\}}\left(\liminf _{\varepsilon \rightarrow 0} \varepsilon \log \sigma_{\varepsilon}(G)\right) \\
& =-\inf _{\{G \in \mathcal{B}: \mu \in G\}}\left(\limsup _{\varepsilon \rightarrow 0} \varepsilon \log \sigma_{\varepsilon}(G)\right) .
\end{aligned}
$$

Remark 5.5. Assuming Theorem 5.1, this shows that, starting with a strong Bernstein-Markov measure $\nu$ and the corresponding sequence of probability measures $\left\{\sigma_{n}\right\}$ on $\mathcal{M}_{P}(K)$ in (5.1), the existence of an LDP with rate function $\mathcal{I}(\mu)$ and speed $2 l_{n}$ implies that necessarily

$$
\mathcal{I}(\mu)=\log J^{Q}\left(\mu_{K, Q}\right)-\log J^{Q}(\mu) .
$$

Uniqueness of the rate function is basic (cf., Lemma 4.1.4 of [13]).

We turn to the first proof of Theorem 5.1, using Theorem 4.5, which gives a pluripotential theoretic description of the rate functional. 
Proof. As a base $\mathcal{B}$ for the topology of $\mathcal{M}_{P}(K)$, we can take the sets from (3.16) or simply all open sets. For $\left\{\sigma_{\varepsilon}\right\}$, we take the sequence of probability measures $\left\{\sigma_{n}\right\}$ on $\mathcal{M}_{P}(K)$ and we take $\varepsilon=\frac{1}{2 l_{n}}$. For $G \in \mathcal{B}$, from (5.2),

$$
\frac{1}{2 l_{n}} \log \sigma_{n}(G)=\log J_{n}^{Q}(G)-\frac{1}{2 l_{n}} \log Z_{n} .
$$

From Proposition 3.7, and (4.14) with $v=Q$,

$$
\lim _{n \rightarrow \infty} \frac{1}{2 l_{n}} \log Z_{n}=\log \delta^{Q}(K)=\log J^{Q}\left(\mu_{K, Q}\right) ;
$$

and by Theorem 4.5,

$$
\inf _{G \ni \mu} \limsup _{n \rightarrow \infty} \log J_{n}^{Q}(G)=\inf _{G \ni \mu} \liminf _{n \rightarrow \infty} \log J_{n}^{Q}(G)=\log J^{Q}(\mu) .
$$

Thus by Proposition $5.3\left\{\sigma_{n}\right\}$ satisfies an LDP with rate function

$$
\mathcal{I}(\mu):=\log J^{Q}\left(\mu_{K, Q}\right)-\log J^{Q}(\mu)
$$

and speed $2 l_{n}$. This rate function is good since $\mathcal{M}_{P}(K)$ is compact.

Remark 5.6. From Proposition 4.7, $\mu_{K, Q}$ is the unique maximizer of the functional

$$
\mu \longrightarrow \log J^{Q}(\mu)
$$

over all $\mu \in \mathcal{M}_{P}(K)$. Thus

$$
\mathcal{I}_{K, Q}(\mu) \geq 0 \text { with } \mathcal{I}_{K, Q}(\mu)=0 \quad \Longleftrightarrow \quad \mu=\mu_{K, Q}
$$

To summarize, $\mathcal{I}_{K, Q}$ is a good rate function with unique minimizer $\mu_{K, Q}$. Using the relations

$$
\begin{aligned}
\log J(\mu) & =-b_{d} \sup _{Q \in C(K)}\left[E\left(V_{P, K, Q}^{*}\right)-\int_{K} Q d \mu\right] \\
J(\mu) & =J^{Q}(\mu) \cdot\left(e^{\int_{K} Q d \mu}\right)^{b_{d}}, \text { and } J^{Q}\left(\mu_{K, Q}\right)=\delta^{Q}(K)
\end{aligned}
$$

(the latter from (4.19)), we have

$$
\begin{aligned}
\mathcal{I}(\mu) & :=\log \delta^{Q}(K)-\log J^{Q}(\mu) \\
& =\log \delta^{Q}(K)-\log J(\mu)+b_{d} \int_{K} Q d \mu \\
& =b_{d} \sup _{Q \in C(K)}\left[E\left(V_{P, K, Q}^{*}\right)-\int_{K} Q d \mu\right]+\log \delta^{Q}(K)+b_{d} \int_{K} Q d \mu \\
& =b_{d} \sup _{v \in C(K)}\left[E\left(V_{P, K, v}^{*}\right)-\int_{K} v d \mu\right]-b_{d}\left[E\left(V_{P, K, Q}^{*}\right)-\int_{K} Q d \mu\right]
\end{aligned}
$$

from (4.6). 
The second proof of our LDP follows from Corollary 4.6.14 in [13], which is a general version of the Gärtner-Ellis theorem. This approach was originally brought to our attention by S. Boucksom and was also utilized by R. Berman in [5]. We state the version of the [13] result for an appropriate family of probability measures.

Proposition 5.7. Let $C(K)^{*}$ be the topological dual of $C(K)$, and let $\left\{\sigma_{\varepsilon}\right\}$ be a family of probability measures on $\mathcal{M}_{P}(K) \subset C(K)^{*}$ (equipped with the weak-* topology). Suppose for each $\lambda \in C(K)$, the limit

$$
\Lambda(\lambda):=\lim _{\varepsilon \rightarrow 0} \varepsilon \log \int_{C(K)^{*}} e^{\lambda(x) / \varepsilon} d \sigma_{\varepsilon}(x)
$$

exists as a finite real number and assume $\Lambda$ is Gâteaux differentiable; i.e., for each $\lambda, \theta \in C(K)$, the function $f(t):=\Lambda(\lambda+t \theta)$ is differentiable at $t=0$. Then $\left\{\sigma_{\varepsilon}\right\}$ satisfies an $L D P$ in $C(K)^{*}$ with the convex, good rate function $\Lambda^{*}$.

Here

$$
\Lambda^{*}(x):=\sup _{\lambda \in C(K)}(<\lambda, x>-\Lambda(\lambda)),
$$

is the Legendre transform of $\Lambda$. The upper bound (5.5) in the LDP holds with rate function $\Lambda^{*}$ under the assumption that the limit $\Lambda(\lambda)$ exists and is finite; the Gâteaux differentiability of $\Lambda$ is needed for the lower bound (5.4). To verify this property in our setting, we must recall a result from [2].

Proposition 5.8. For $Q \in \mathcal{A}(K)$ and $u \in C(K)$, let

$$
F(t):=E\left(V_{P, K, Q+t u}^{*}\right)
$$

for $t \in \mathbb{R}$. Then $F$ is differentiable and

$$
F^{\prime}(t)=\int_{\mathbb{C}^{d}} u\left(d d^{c} V_{P, K, Q+t u}^{*}\right)^{d} .
$$

In [2] it was assumed that $u \in C^{2}(K)$ but the result is true with the weaker assumption $u \in C(K)$ (cf., Theorem 11.11 in [16] due to Lu and Nguyen [17], see also [11, Proposition 4.20]).

We proceed with the second proof of Theorem 5.1. For simplicity, we normalize so that $\gamma_{d}=1$ to fit the setting of Proposition 5.7 (so members of $\mathcal{M}_{P}(K)$ are probability measures).

Proof. We show that for each $v \in C(K)$,

$$
\Lambda(v):=\lim _{n \rightarrow \infty} \frac{1}{2 l_{n}} \log \int_{C(K)^{*}} e^{2 l_{n}<v, \mu>} d \sigma_{n}(\mu)
$$


exists as a finite real number. First, since $\sigma_{n}$ is a measure on $\mathcal{M}_{P}(K)$, the integral can be taken over $\mathcal{M}_{P}(K)$. Consider

$$
\frac{1}{2 l_{n}} \log \int_{\mathcal{M}_{P}(K)} e^{2 l_{n}<v, \mu>} d \sigma_{n}(\mu) .
$$

By (5.3), this is equal to

$$
\frac{1}{2 l_{n}} \log \frac{1}{Z_{n}} \cdot \int_{K^{d_{n}}}\left|V D M_{n}^{Q-\frac{l_{n}}{n d_{n}} v}\left(x_{1}, \ldots, x_{d_{n}}\right)\right|^{2} d \nu\left(x_{1}\right) \ldots d \nu\left(x_{d_{n}}\right) .
$$

From (4.5), with $\gamma_{d}=1, \frac{l_{n}}{n d_{n}} \rightarrow \frac{1}{b_{d}}$; hence for any $\varepsilon>0$,

$$
\frac{1}{b_{d}+\varepsilon} v \leq \frac{l_{n}}{n d_{n}} v \leq \frac{1}{b_{d}-\varepsilon} v \text { on } K
$$

for $n$ sufficiently large. Recall that

$$
\left.Z_{n}=\int_{K^{d_{n}}} \mid V D M_{n}^{Q}\left(x_{1}, \ldots, x_{d_{n}}\right)\right)\left.\right|^{2} d \nu\left(x_{1}\right) \ldots d \nu\left(x_{d_{n}}\right) .
$$

Define

$$
\widetilde{Z}_{n}:=\int_{K^{d_{n}}}\left|V D M_{n}^{Q-v / b_{d}}\left(x_{1}, \ldots, x_{d_{n}}\right)\right|^{2} d \nu\left(x_{1}\right) \ldots d \nu\left(x_{d_{n}}\right) .
$$

Then we have

$$
\lim _{n \rightarrow \infty} \widetilde{Z}_{n}^{\frac{1}{2 l_{n}}}=\delta^{Q-v / b_{d}}(K) \text { and } \lim _{n \rightarrow \infty} Z_{n}^{\frac{1}{2 l_{n}}}=\delta^{Q}(K)
$$

from (3.15) in Proposition 3.7 and the assumption that $(K, \nu, \widetilde{Q})$ satisfies the weighted Bernstein-Markov property for all $\widetilde{Q} \in C(K)$. Thus

$$
\Lambda(v)=\lim _{n \rightarrow \infty} \frac{1}{2 l_{n}} \log \frac{\widetilde{Z}_{n}}{Z_{n}}=\log \frac{\delta^{Q-v / b_{d}}(K)}{\delta^{Q}(K)} .
$$

Define now, for $v, v^{\prime} \in C(K)$,

$$
f(t):=E\left(V_{P, K, Q-\left(v+t v^{\prime}\right)}^{*}\right) .
$$

Proposition 5.8 shows that $\Lambda$ is Gâteaux differentiable and Proposition 5.7 gives that $\Lambda^{*}$ is a rate function on $C(K)^{*}$.

Since each $\sigma_{n}$ has support in $\mathcal{M}_{P}(K)$, it follows from (5.4) and (5.5) in Definition 5.2 of an LDP with $\Gamma \subset C(K)^{*}$ that for $\mu \in C(K)^{*} \backslash \mathcal{M}_{P}(K), \Lambda^{*}(\mu)=+\infty$. By Lemma 4.1 .5 (b) of [13], the restriction of $\Lambda^{*}$ to $\mathcal{M}_{P}(K)$ is a rate function. Since $\mathcal{M}_{P}(K)$ is compact, it is a good rate function. Being a Legendre transform, $\Lambda^{*}$ is convex. 
To compute $\Lambda^{*}$, we have, using (5.7) and (3.11),

$$
\begin{aligned}
\Lambda^{*}(\mu) & =\sup _{v \in C(K)}\left(\int_{K} v d \mu-\log \frac{\delta^{Q-v / b_{d}}(K)}{\delta^{Q}(K)}\right) \\
& =\sup _{v \in C(K)}\left(\int_{K} v d \mu-b_{d}\left[E\left(V_{P, K, Q}^{*}\right)-E\left(V_{P, K, Q-v / b_{d}}^{*}\right]\right)\right) .
\end{aligned}
$$

Thus

$$
\begin{aligned}
\Lambda^{*}(\mu)+b_{d} E\left(V_{P, K, Q}^{*}\right) & =\sup _{v \in C(K)}\left(\int_{K} v d \mu+b_{d} E\left(V_{P, K, Q-v / b_{d}}^{*}\right)\right) \\
& =\sup _{u \in C(K)}\left(b_{d} E\left(V_{P, K, Q+u}^{*}\right)-b_{d} \int_{K} u d \mu\right)\left(\operatorname{taking} u=-v / b_{d}\right) .
\end{aligned}
$$

Rearranging and replacing $u$ in the supremum by $v=u+Q$,

$$
\begin{aligned}
\Lambda^{*}(\mu) & =\sup _{u \in C(K)}\left(b_{d} E\left(V_{P, K, Q+u}^{*}\right)-b_{d} \int_{K} u d \mu\right)-b_{d} E\left(V_{P, K, Q}^{*}\right) \\
& =b_{d}\left[\sup _{v \in C(K)} E\left(V_{P, K, v}^{*}\right)-\int_{K} v d \mu\right]-b_{d}\left[E\left(V_{P, K, Q}^{*}\right)-\int_{K} Q d \mu\right]
\end{aligned}
$$

which agrees with the formula in Remark 5.6 (since $\mu$ is a probability measure).

Remark 5.9. Thus the rate function can be expressed in several equivalent ways:

$$
\begin{aligned}
\mathcal{I}(\mu) & =\Lambda^{*}(\mu)=\log J^{Q}\left(\mu_{K, Q}\right)-\log J^{Q}(\mu) \\
& =b_{d}\left[\sup _{v \in C(K)} E\left(V_{P, K, v}^{*}\right)-\int_{K} v d \mu\right]-b_{d}\left[E\left(V_{P, K, Q}^{*}\right)-\int_{K} Q d \mu\right] \\
& =b_{d} E^{*}(\mu)-b_{d}\left[E\left(V_{P, K, Q}^{*}\right)-\int_{K} Q d \mu\right]
\end{aligned}
$$

which generalizes the result equating (5.3), (5.10) and (5.11) in [9] for the case $P=\Sigma$ and $b_{d}=1$. Note in the last equality we are using the slightly different notion of $E^{*}$ in (2.9) and Proposition 3.4 than that used in [9].

\section{References}

1. Bayraktar, T., Zero distribution of random sparse polynomials, Michigan Math. J. 66 (2017), 389-419. MR3657224

2. Bayraktar, T., Bloom, T. and Levenberg, N., Pluripotential theory and convex bodies, Mat. Sb. 209 (2018), 67-101. MR3769215 
3. Bedford, E. and Taylor, B. A., The Dirichlet problem for a complex Monge-Ampère equation, Invent. Math. 37 (1976), 1-44. MR0445006

4. Bedford, E. and TAYLOR, B. A., A new capacity for plurisubharmonic functions, Acta Math. 149 (1982), 1-40. MR0674165

5. Berman, R., Determinantal Point Processes and Fermions on Complex Manifolds: Large Deviations and Bosonization, Comm. Math. Phys. 327 (2014), 147. MR3177931

6. Berman, R. and Boucksom, S., Growth of balls of holomorphic sections and energy at equilibrium, Invent. Math. 181 (2010), 337-394. MR2657428

7. Berman, R., Boucksom, S., Guedu, V. and Zeriahi, A., A variational approach to complex Monge-Ampère equations, Publ. Math. Inst. Hautes Études Sci. 117 (2013), 179-245. MR3090260

8. Bloom, T. and Levenberg, N., Pluripotential energy, Potential Anal. 36 (2012), 155-176. MR2886457

9. Bloom, T. and Levenberg, N., Pluripotential energy and large deviation, Indiana Univ. Math. J. 62 (2013), 523-550. MR3158519

10. Boucksom, S., Eyssidieux, P., Guedu, V. and Zeriahi, A., Monge-Ampère equations in big cohomology classes, Acta Math. 205 (2010), 199-262. MR2746347

11. Darvas, T., Di Nezza, E. and Lu, C. H., Monotonicity of nonpluripolar products and complex Monge-Ampère equations with prescribed singularity, Anal. PDE 11 (2018), 2049-2087. MR3812864

12. Darvas, T., Di Nezza, E. and Lu, C. H., Log-concavity of volume and complex Monge-Ampère equations with prescribed singularity. arXiv : 1807.00276.

13. Dembo, A. and Zeitouni, O., Large deviations techniques and applications, Jones and Bartlett Publishers, Boston, MA, 1993. MR1202429

14. Guedu, V. and Zeriahi, A., Intrinsic capacities on compact Kähler manifolds, J. Geom. Anal. 15 (2005), 607-639. MR2203165

15. Guedj, V. and Zeriahi, A., The weighted Monge-Ampère energy of quasiplurisubharmonic functions, J. Funct. Anal. 250 (2007), 442-482. MR2352488

16. Guedu, V. and Zeriahi, A., Degenerate Complex Monge-Ampère Equations, European Math. Soc. Tracts in Mathematics 26, 2017. MR3617346

17. Lu, C. H. and NGuyen, V. D., Degenerate complex Hessian equations on compact Kähler manifolds, Indiana Univ. Math. J. 64 (2015), 1721-1745. MR3436233

18. Ross, J. and Nyström, D. W., Analytic test configurations and geodesic rays, J. Symplectic Geom. 12 (2014), 125-169. MR3194078

19. SAfF, E. and Tотік, V., Logarithmic potentials with external fields, Springer, Berlin, 1997. MR1485778

20. Nyström, D. W., Monotonicity of nonpluripolar Monge-Ampère masses, Indiana Univ. Math. J. 68 (2019) 579-591. MR3951074

Turgay Bayraktar

Sabanci University

Istanbul 34956

Turkey

tbayraktar@sabanciuniv.edu
Thomas Bloom

University of Toronto

Toronto

Ontario M5S 2E4

Canada

bloom@math.toronto.edu 
Norman Levenberg

Indiana University

Bloomington

IN 47405

USA

nlevenbe@indiana.edu

Received August 17, 2018

in revised form February 10, 2019
Chinh H. Lu

Université Paris-Sud

Orsay 91405

France

hoang-chinh.lu@u-psud.fr 